\title{
Géolinguistique
}

$14 \mid 2013$

Varia

\section{Météoronymes dans la province de Tarente}

Meteoronyms in the Province of Taranto

Carlotta D'Addario

\section{OpenEdition}

Journals

Édition électronique

URL : http://journals.openedition.org/geolinguistique/860

DOI : $10.4000 /$ geolinguistique. 860

ISSN : 2650-8176

\section{Éditeur}

UGA Éditions/Université Grenoble Alpes

\section{Édition imprimée}

Date de publication : 15 décembre 2013

Pagination : 89-123

ISBN : 978-2-84310-264-6

ISSN : 0761-9081

\section{Référence électronique}

Carlotta D'Addario, " Météoronymes dans la province de Tarente », Géolinguistique [En ligne], 14 | 2013, mis en ligne le 30 avril 2019, consulté le 02 novembre 2020. URL : http://journals.openedition.org/ geolinguistique/860 ; DOI : https://doi.org/10.4000/geolinguistique.860 


\title{
Météoronymes dans la province de Tarente
}

\author{
Carlotta D'Addario \\ Université de Turin
}

\section{Résumé}

Notre recherche porte sur l'analyse dans une perspective diachronique des dénominations météorologiques dans la province de Tarente (Italie), en relation avec les nouvelles conditions sociales et économiques de la région. Deux facteurs principaux sont pris en compte dans cette étude : 1) l'influence de la langue italienne sur la production linguistique des informateurs ; 2) les interférences liées au questionnaire utilisé.

Les résultats de cette recherche montrent une réduction et un changement qualitatif des différents types lexicaux, par rapport à ceux enregistrés dans le passé. La langue italienne remplace les formes locales de «concepts généraux» (froid, chaud), alors que, dans le cas de noms de «concepts graduels » (bruine, pluie), l'italien agit indirectement en déconstruisant le domaine de la variation lexicale : les formes dialectales sont italianisées ou cèdent la place à des périphrases génériques. Le dialecte perd sa capacité de créer de nouvelles métaphores. La province de Tarente montre des traits bien distincts d'italianisation dans le champ lexical, en particulier concernant les conditions météorologiques. Le répertoire linguistique de l'aire étudiée pourrait être considérée comme divisée en trois domaines : 1) la norme italienne; 2) le dialecte local; 3) au milieu, un grand domaine constitué de phénomènes dont la catégorisation est difficile, étant caractérisé par la polysémie, l’hypertrophie et l'imprécision sémantique.

\section{Mots-clés}

Géolinguistique, météoronymie, dialectes italo-romans. 


\begin{abstract}
Our research focuses on the analysis through a diachronic perspective of meteorological names in the province of Taranto (Italy), in relation to the new social and economic conditions of the area. Two main factors are considered in this study: 1) the influence of the Italian language on the linguistic production of the informants; 2) the interferences due to the questionnaire employed.

The results of this research show a reduction and a qualitative change of the different lexical types, compared to the ones registered in the past. The Italian language replaces the local forms of "general concepts" (cold, hot), whereas, in the case of names of "gradual concepts" (drizzle, downpour), Italian acts indirectly by deconstructing the field of lexical variation: the dialectal forms are Italianized or give way to generic periphrases. The dialect loses its ability to self-construct metaphors. The province of Taranto shows very distinct traits of Italianization in the lexical field, particularly concerning meteorological terms. The linguistic repertoire of the area investigated could be seen as divided in three domains: 1) the Italian standard; 2) the local dialect; 3) in the middle, a large domain constituted by phenomena whose categorization is difficult, as it is characterized by polysemy, hypertrophy and semantic imprecision.
\end{abstract}

\title{
Keywords
}

Linguistic geography, meteoronymy, italo-romance dialects.

En partant du concept que la vitalité linguistique se présente comme le miroir du «sentimento di immediata comunione dell'individuo con il suo ambiente» (Terracini, 1970, p. 178), il est nécessaire d'effectuer une analyse des phénomènes de cohésion ou désagrégation lexicale qui considère à la fois les capacités réactives du système et le rôle de chaque locuteur. En effet, d'après Terracini (1970), notre histoire linguistique par le biais des «vicende dei vocaboli passati da individuo ad individuo, da ambiente ad ambiente, riflette propriamente la storia della fortuna delle idee, di concezioni di forme di vita che una comunità viene man mano accogliendo e svolgendo» (p. 178). Pour cette raison, le point de départ dans l'analyse du lexique doit donc être représenté par la relation entre le locuteur et son milieu.

La vitalité de la langue parlée implique que le lexique d'une langue vivante ne puisse être homogène : elle a tendance à se manifester de façon multiple dans les différentes couches du lexique. Par exemple, d'après Lazzari (1919), «certe idee cambiano denominazione secondo i tempi e 
i luoghi per ragioni dipendenti da ambiente; ad es. i nomi di 'casa vesti suppellettili', etc.; altre di carattere generale, che non dipendono tanto dall'arbitrio degli uomini restano salde nelle denominazioni; ad esempio i nomi di 'numeri-padre-madre', etc.» (p. 2-3). Cela laisse conclure que, comme l'on verra plus loin, quant aux météoronymes il existe une différence entre les phénomènes atmosphériques concernant des concepts généraux (comme pluie), qui présentent une uniformité de dénomination, et les concepts (comme bruine) qui montrent normalement, dans les dialectes, une grande variété de dénominations.

Les réflexions évoquées ci-dessus sont à l'origine des recherches menées, leur objet d'étude étant constitué par les dénominations des météoronymes dans la province de Tarente. Les données analysées, avec une approche comparative, sont d'une part les données enregistrées au cours des quatre-vingts dernières années dans AIS, ALI, VDS et Seminario $2006^{1}$, concernant les dénominations de certains phénomènes atmosphériques (à la frontière entre les Pouilles et le Salento), et d'autre part, les données collectées par nos soins lors de l'étude Inchieste 2008. Les points d'enquête ont été choisis dans la province de Tarente. Cinq d'entre eux se situent dans la zone nord-occidentale de la province et cinq autres appartiennent à la zone sud-est : Ginosa, Castellaneta, Palagianello, Crispiano, Statte, Talsano, Pulsano, Lizzano, Sava et Maruggio.

Parmi les objectifs de notre recherche : analyse, en diachronie, des dénominations de certains phénomènes atmosphériques, compte tenu aussi des changements des conditions économiques au fil du temps, à l'intérieur de la zone d'enquête; étude de l'interférence entre l'italien et le dialecte; analyse des phénomènes d'interférence causés par la technique de traduction du questionnaire.

Le questionnaire se composait de 28 phrases qui contenaient 34 météoronymes et l'échantillon choisi était de 100 informateurs, 10 pour chaque point dont 5 âgés de 18 à 30 ans avec un niveau de scolarité correspondant au baccalauréat et 5 âgés de plus de 60 ans avec un niveau de scolarité correspondant au diplôme élémentaire. De plus, une des conditions requises était d'être né et d'avoir toujours vécu dans le village. Parmi tous les météoronymes analysés, seuls les plus marquants seront cités.

1. Séminaire de dialectologie italienne qui a eu lieu auprès de la Faculté des langues et littératures étrangères de l'Université du Salento au printemps 2006 sous la direction du professeur Alberto A. Sobrero. 


\section{Rosée, givre, gel}

À l'intérieur des dénominations des météoronymes dans la province de Tarente, un des cas les plus intéressants est représenté par les dénominations de rosée, givre et gel.

Le dictionnaire Zingarelli s.v. rosée (lat. ros, roris) indique : «deposito su oggetti al suolo di goccioline d'acqua provenienti dalla condensazione del vapore acqueo contenuto nell'aria» (Zingarelli, 2005); s.v. givre (lat. pruina) : «deposito di cristalli di ghiaccio che si forma per sublimazione nelle notti serene su oggetti con temperatura inferiore a zero gradi» (ibid.); s.v. gel (lat. gĕlu), enfin : «ghiaccio, brinata, gelata» (ibid.).

À partir d'une vue d'ensemble sur l'Italie dialectale, avec Lazzari on constate que, en ce qui concerne les dénominations de la rosée, il y a notamment :

1) type lexical rugiada 'rosée' dans les dialectes d'Italie du Nord (terme peu fréquent dans les dialectes centro-méridionaux);

2) dérivés du terme acqua 'eau' avec différents suffixes dans les dialectes centro-méridionaux (Lazzari, 1919, p. 67).

En revanche, en ce qui concerne le terme givre, Lazzari observe que le mot latin pruina a survécu seulement dans quelques dialectes; pour la plupart, il y a :

1) terme italien brina 'givre' dans les dialectes gallo-italiques;

2) dérivés des termes brina 'givre', ghiaccio 'glace' et acqua 'eau' dans les dialectes des régions du centre et du sud (Lazzari, 1919, p. 71-72).

De plus, Lazzari a constaté que le météoronyme en question se présente sous différentes formes selon l'endroit et le climat dans lequel il se produit. Bien évidemment, ces formes ne sont pas clairement distinguées par les locuteurs et les différentes dénominations populaires (qui ne présentent pas toujours une correspondance en italien) peuvent exprimer des phénomènes propres à chaque région (Lazzari, 1919, p. 71-72).

Concernant la province de Tarente, dans l'AIS nous retrouvons une distinction lexicale claire entre la rosée et le givre/le gel. Pour le terme rosée (c. 374) on enregistre les dérivés du terme acqua 'eau' à Palagiano et Avetrana, tandis que pour le terme givre (c.375) on indique le type lexical ferratura à Palagiano et le type šilatura à Avetrana. Pour le terme gel on observe les types lexicaux šilatura/farratura à Palagiano et le seul type šilatura à Avetrana. 
Dans l'ALI, toujours pour la province de Tarente, sous le mot rosée, on observe acquaggho dans la partie nord-occidentale de la province et plus précisément à Laterza, Palagianello, Crispiano et Tarente; quant à Manduria, le type endémique muttori est enregistré. Gel et givre sont cités dans l'ALI, pour tous les points d'enquête, avec le même type lexical. Le type scialatura est indiqué en plusieurs variantes à Laterza, Palagianello, Crispiano et Tarente, hormis Manduria où l'on trouve scilazza.

Dans le VDS, concernant la province de Tarente, pour le mot rosée, les dérivés du terme acqua 'eau' avec différents suffixes sont signalés à Tarente, Martina Franca, Massafra, Palagiano et Avetrana; en outre, le type lexical muttòre (du verbe muttare : 'mouiller, tremper') dans différentes variantes phonétiques à Manduria, Uggiano Montefusco et Sava. Pour le mot givre on compte le type chiatrore à Tarente et le type scilatura en plusieurs variantes phonétiques à Avetrana, Grottaglie, Massafra, Martina Franca, Manduria et Sava. Pour le terme gel, le VDS indique aussi les mêmes résultats que pour givre, avec seulement quelques différences dans la distribution géographique.

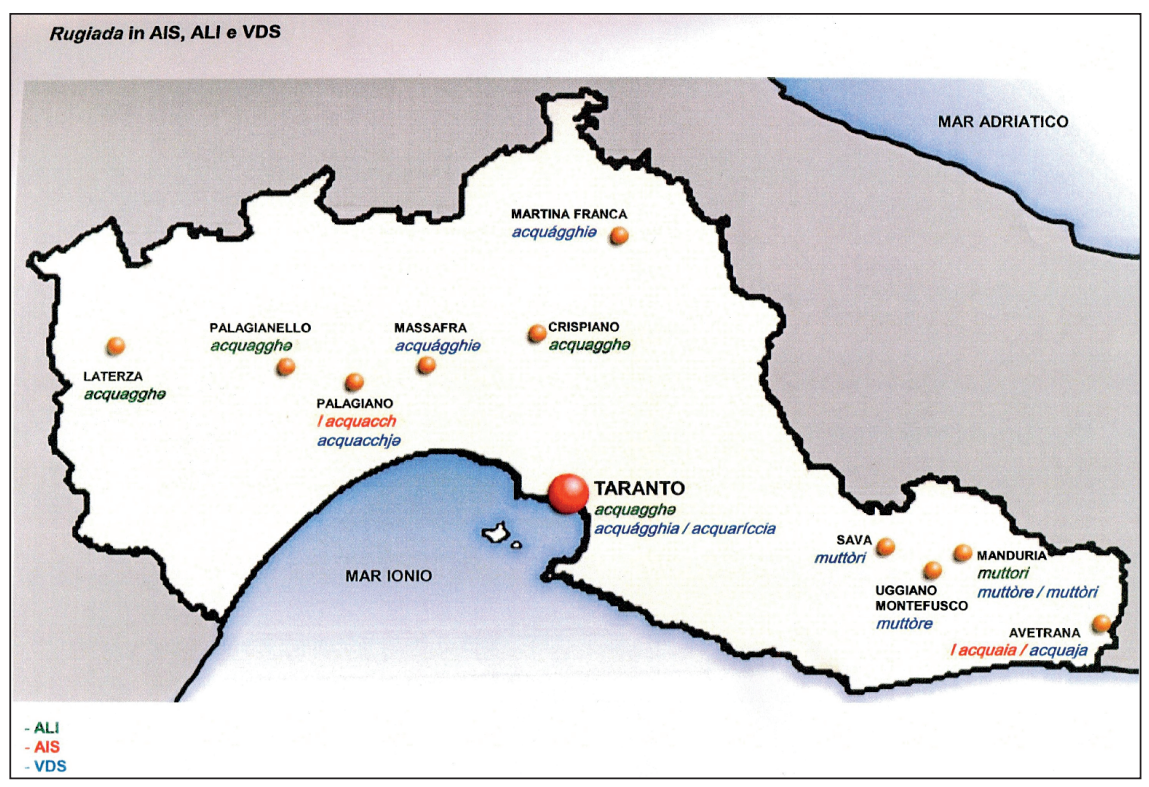



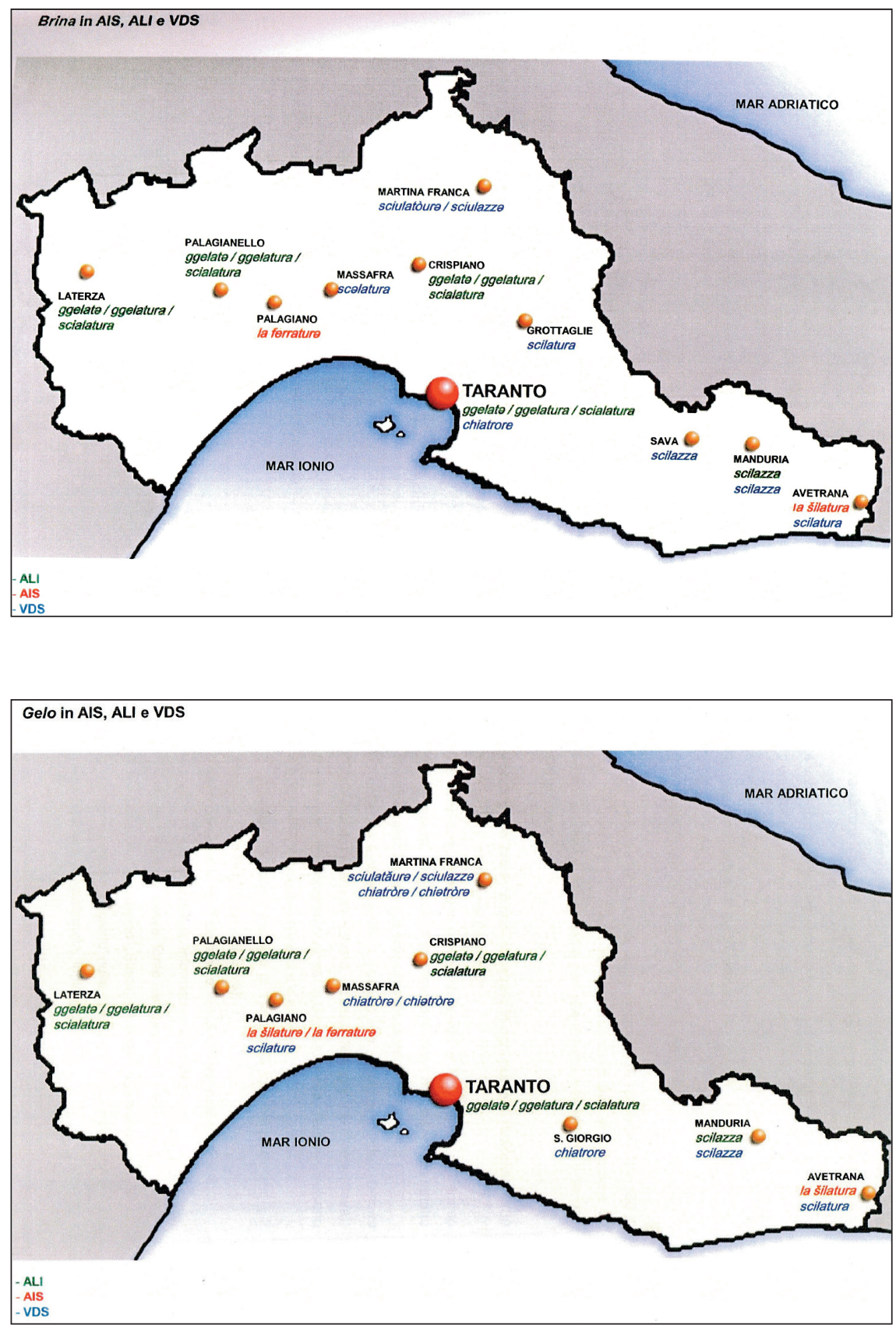
En comparant ces données avec les données recueillies dans la province de Tarente pour le Seminario 2006 et les Inchieste 2008 on remarque que, après un peu plus d'un demi-siècle, l'opposition rosée v/s givre - gel est de moins en moins ressentie. Les trois phénomènes atmosphériques ont tendance à être confondus, perçus comme la même chose; des lexèmes à signification spécifique sont souvent remplacés par d'autres qui appartiennent au même champ sémantique.

Pour le mot givre, on peut observer que dans toute la zone analysée, la forme italienne, la plus courante, est en train de prendre le dessus. Ensuite, pour le nombre d'occurrences, on retrouve le type lexical ak:wag:ja enregistré presque exclusivement dans la zone nord-occidentale de la province.

En outre, sur l'ensemble du territoire une superposition/confusion de plusieurs termes est remarquée : les variantes du type lexical filatura (Castellaneta, Statte, Pulsano, Lizzano, Maruggio et Sava) et le type italien dz:elo (Ginosa, Statte, Talsano, Pulsano et Lizzano) sont distribués sur toute la zone, ces deux alternent avec quelques occurrences du type endémique kjatrore ou avec des hapax tels que gjat:a 'glace', neb:ja 'brouillard', mut:ore, freskura et rudz:ada 'rosée'. Dans la zone située au sud-est de Tarente, enfin, la traduction du type 'um:uto (5 occurrences) est signalée.

Pour citer Terracini (1970), on pourrait dire que «l'accavallarsi di correnti svariate che premono su un 'patois en détresse' porta con sé una singolare concorrenza di sinonimi ed una incertezza sul significato di ciascuno di essi $[\ldots] »$ (p. 97).

À partir de cela, il est possible de remarquer comment les types lexicaux qui désignaient dans le passé la rosée viennent aujourd'hui indiquer un phénomène différent. C'est le cas pour le type lexical ak:wag:ja : dans AIS, ALI et VDS, le terme est enregistré en tant que traduction du vocable rosée alors que dans Seminario 2006 et Inchieste 2008 il apparaît plusieurs fois comme la traduction de givre.

Le même phénomène, quoique moins évident, se produit pour le type lexical 'um:uto (donnée relevée pour 3 informateurs jeunes et 2 âgés au sud-est de Tarente) : le VDS indique le terme en tant que traduction de rosée à Latiano et Carovigno, tandis que pour 5 interviews ici conduites, il est enregistré en tant que traduction de givre. Cela vaut également pour l'occurrence du type rudz:ada (italien) et pour le type mut:ore (type endémique inscrit dans VDS et ALI en tant que traduction de rosée dans la zone sud-orientale de la province).

Enfin, il est à noter que dans Inchieste 2008 le type lexical ferraturo n'est pas enregistré (attesté seulement dans l'AIS à Palagiano) et il est donc possible de supposer qu'il s'agit d'une forme archaïque désormais abandonnée. Pour ce qui est du type endémique chiatrore, mentionné dans 
VDS en tant que traduction de givre et gel dans une zone assez élargie, il est encore signalé à Crispiano et à Pulsano dans un nombre limité d'occurrences (2) de la part d'informateurs âgés de plus de 60 ans : il est donc possible de supposer que ce type lexical soit considéré archaïque et probablement destiné à disparaître.

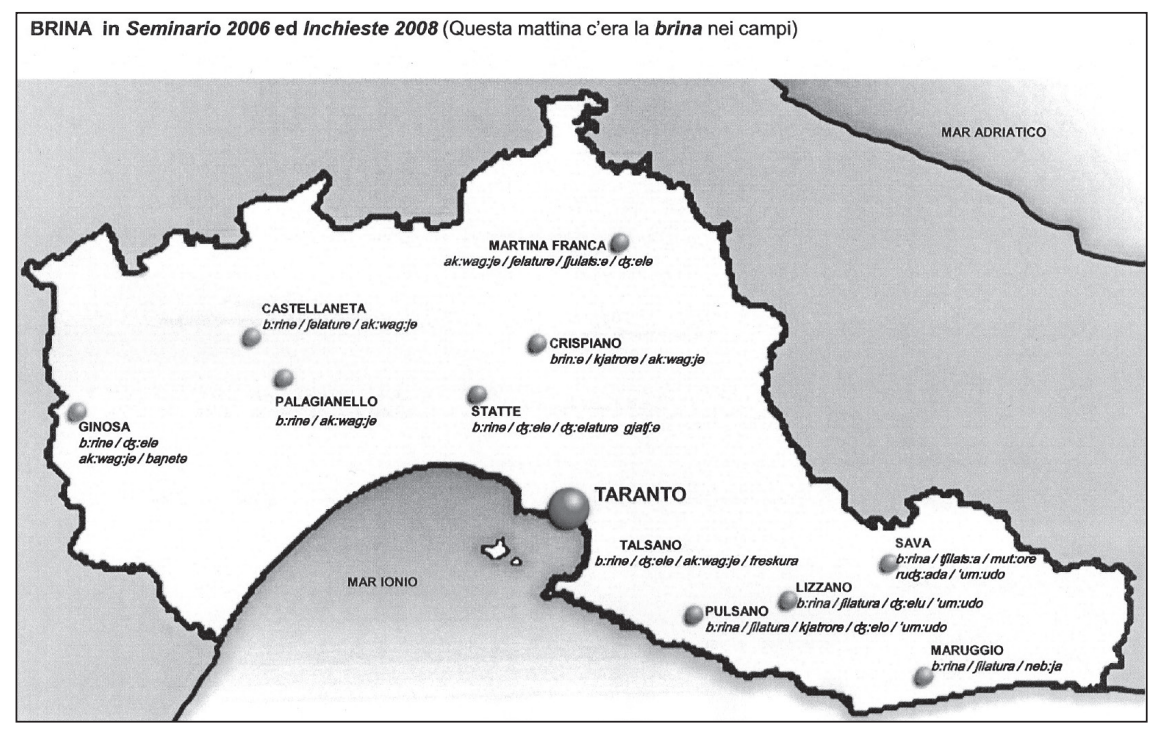

À ce stade, les résultats enregistrés pour le mot rosée seront analysés. Outre le type lexical italien, diffusé et enregistré sur tout le territoire, en général nous pouvons constater une prédominance du type lexical ak:wag:ja (22 occurrences) dans la partie nord-occidentale de la province; celui-ci s'oppose aux types endémiques filatura et 'um:ido (10 et 12 occurrences) dans la zone sud-orientale de Tarente, où ils se superposent et entrent en contact/conflit.

En évaluant les traductions de rosée, l'impression que les concepts de rosée et de givre ne soient plus distingués par les locuteurs est confirmée. En effet, même dans ce cas, pour la traduction de rosée sont souvent employés les termes utilisés autrefois pour désigner le givre et le gel comme dans le cas des types filatura e kjatrore.

Par rapport à ce qui est enregistré dans AIS, ALI et VDS, nous pouvons remarquer que les dérivés du mot acqua 'eau' ont été bien conservés (déjà identifiés par Lazzari et De Vincentiis en tant que traductions de rosée). Ceux-ci sont aujourd'hui employés par nos informateurs pour désigner le givre. On enregistre le type endémique muttore (signalé dans ALI et VDS en tant que traduction de rosée) pour une seule interview, où il est utilisé 
pour traduire le terme givre et non pas le terme rosée. Le terme appartient au dialecte du Salento et il a été encore signalé lors des interviews réalisées pour le Seminario 2006 dans cette aire. Il est donc légitime de supposer que Sava soit aujourd'hui une des ramifications les plus septentrionales du type lexical en question.

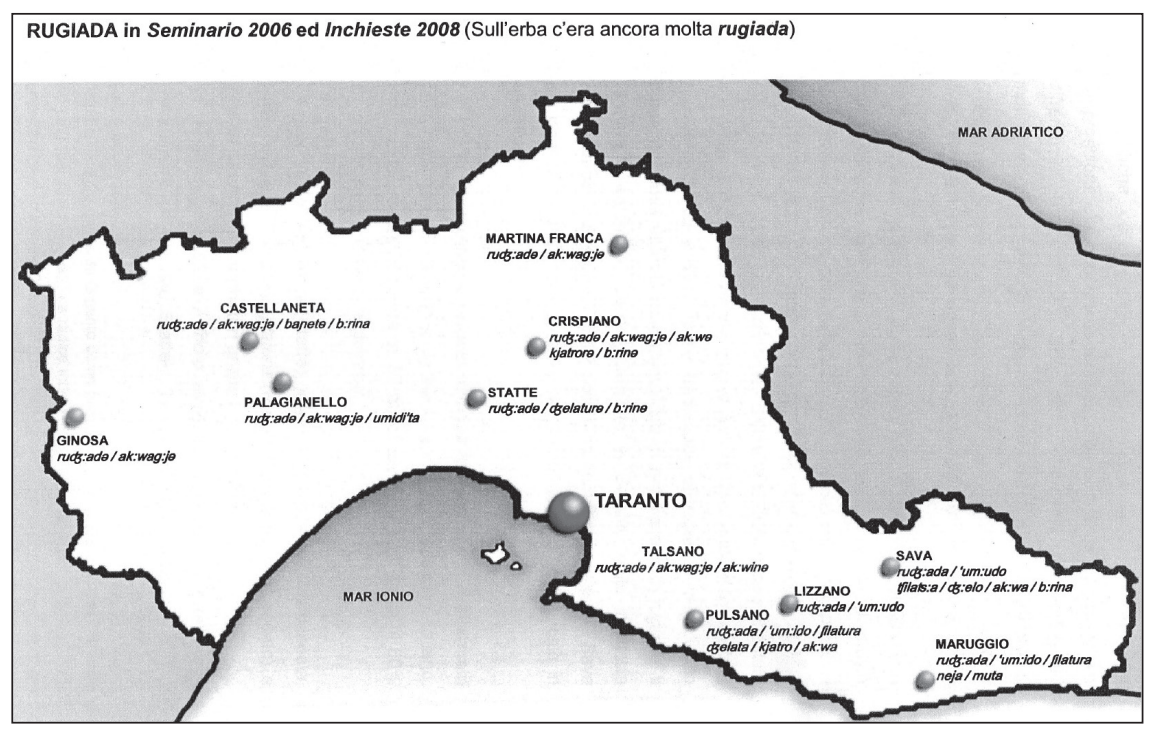

Enfin, en ce qui concerne le gel, le terme qui s'est imposé dans la province de Tarente est ds:elo, type lexical italien, avec des variations phonétiques habituelles dans la zone : 57 occurrences sur 100 dans Inchieste 2008 (presque $60 \%$ des interviews). Sauf pour le type italien, le terme le plus fréquemment utilisé dans la traduction de gel semble être le type endémique filatura qui est enregistré dans presque tous les points d'enquête, dans différentes réalisations phonétiques.

Ensuite, le type lexical frid:o 'froid' (terme générique) qui compte 11 occurrences, n'est signalé dans aucune de nos sources bibliographiques en tant que traduction de gel.

Les occurrences qui restent montrent la permanence des superpositions, même si d'une façon moins évidente, aussi bien pour ce qui est du météoronyme $\mathrm{gel}$ : le vocable est traduit, bien que dans quelques cas, par ak:wag:ja 'rosée', brina 'givre' et 'um:udo 'rosée'. Enfin, même s'il n'existe qu'une seule occurrence, on retrouve le type endémique kjatrore (Crispiano, source femme âgée), enregistré aussi lors du Seminario 2006, pour le point d'enquête Talsano (informateur de plus de 60 ans). 


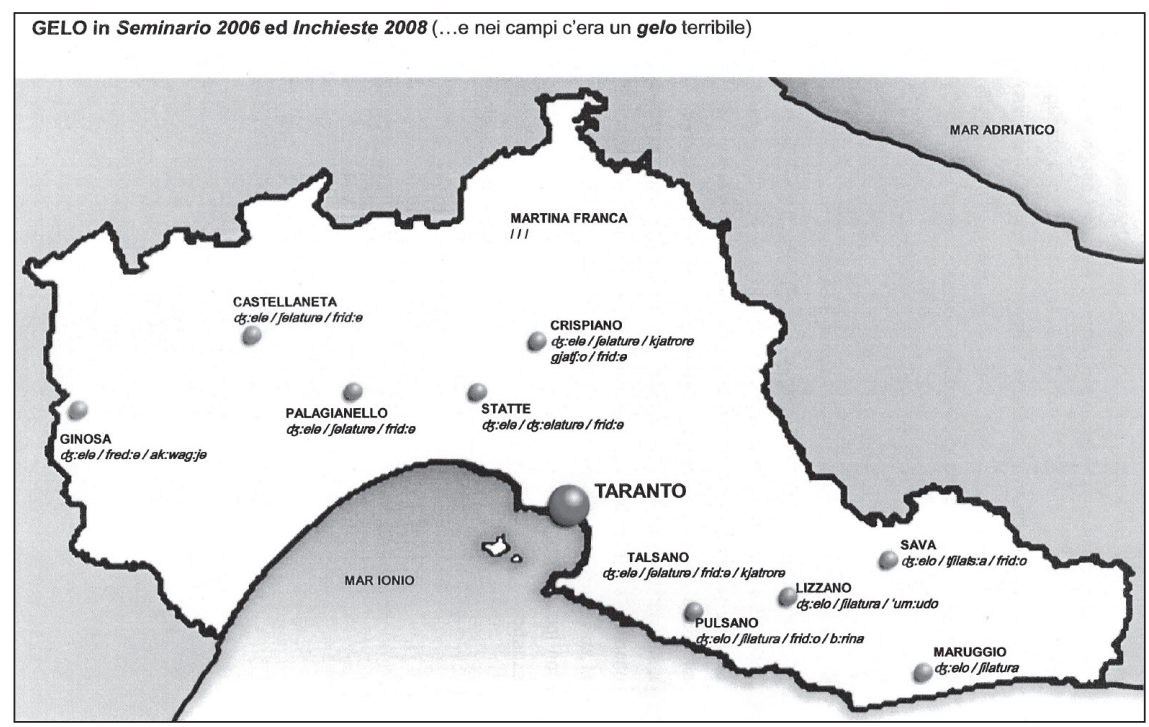

Ce qui a été observé à travers l'analyse des résultats enregistrés pour les mots rosée, givre et gel attire notre attention sur la relation qui existe entre les expressions et les compétences des locuteurs d'une part et la réalité socio-économique de l'autre. L'économie de la province de Tarente, autrefois fondée sur l'agriculture, comme un peu partout dans le sud de l'Italie, s'est aujourd'hui fortement différenciée surtout à travers le développement du secteur tertiaire et de l'industrie, au détriment de l'agriculture. Ceci explique le fait que des concepts tels que la rosée, le givre et le gel, extrêmement importants dans une société agricole, ne soient plus aujourd'hui bien distingués, car ils ont perdu leur raison d'être dans la vie des locuteurs.

\section{Froid, chaud}

Partout dans la province de Tarente et pendant toute la période de temps analysée (environ 80 ans), le froid et le chaud sont toujours nommés avec les mêmes types lexicaux issus des termes latins frīgidus et caldus (syncope de calidus) dans les variantes phonétiques habituelles.

Dans Inchieste 2008 pour l'analyse de froid, les phrases «Ieri ha fatto molto freddo » et «L'inverno è freddo » ont été proposées. Sur 200 réponses, pour 199 fois le type lexical est le type italien, avec des variantes phonétiques dialectales. On enregistre donc le même type lexical répertorié dans AIS et VDS. La seule différence relevée est au niveau phonétique : 
dans le sud-est de la province, de fait, il existe une variante avec voyelle arrière mi-fermée en position finale (frid:o), qui n'est pas attestée dans la bibliographie; il s'agit probablement d'un italianisme. D'ailleurs, le terme fəlèrcə attesté dans VDS à Martina Franca (dans la locution mena fəlèrcə 'il fait froid') n'est pas répertorié dans Inchieste 2008.
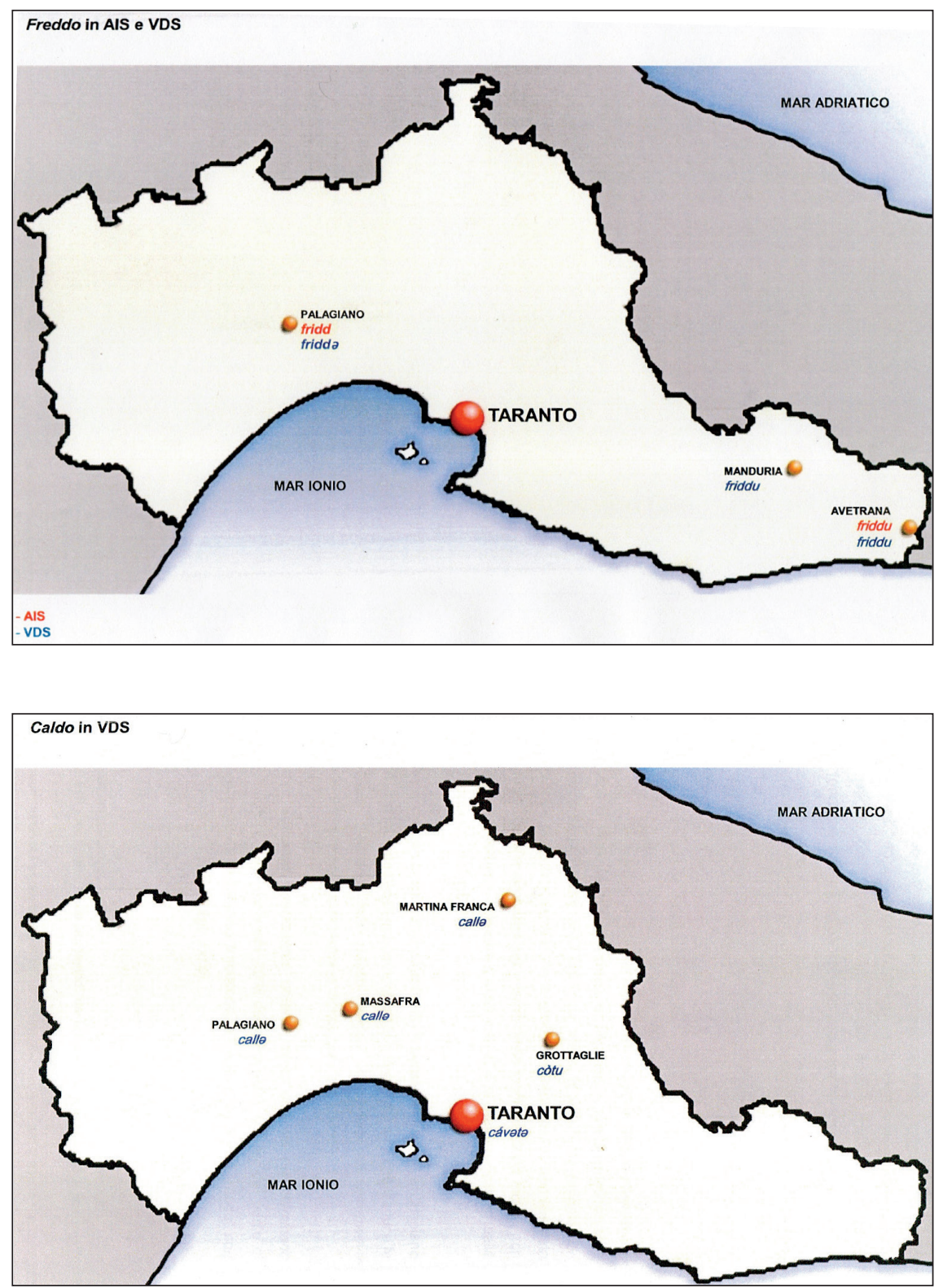
Le seul résultat différent au niveau lexical est attribué à un informateur de Crispiano d'un âge compris entre 18 et 30 ans qui traduit la phrase «Ieri ha fatto molto freddo " par jera sa Đtrenweva (probablement 'hier on tremblait' sous-entendu 'à cause du froid').

Enfin, il est intéressant de remarquer que, aussi bien dans Seminario 2006 que dans Inchieste 2008, deux jeunes informateurs du point d'enquête de Talsano traduisent la phrase «L'inverno è freddo" par l'hiver il fait froid (fajo frid:ə).

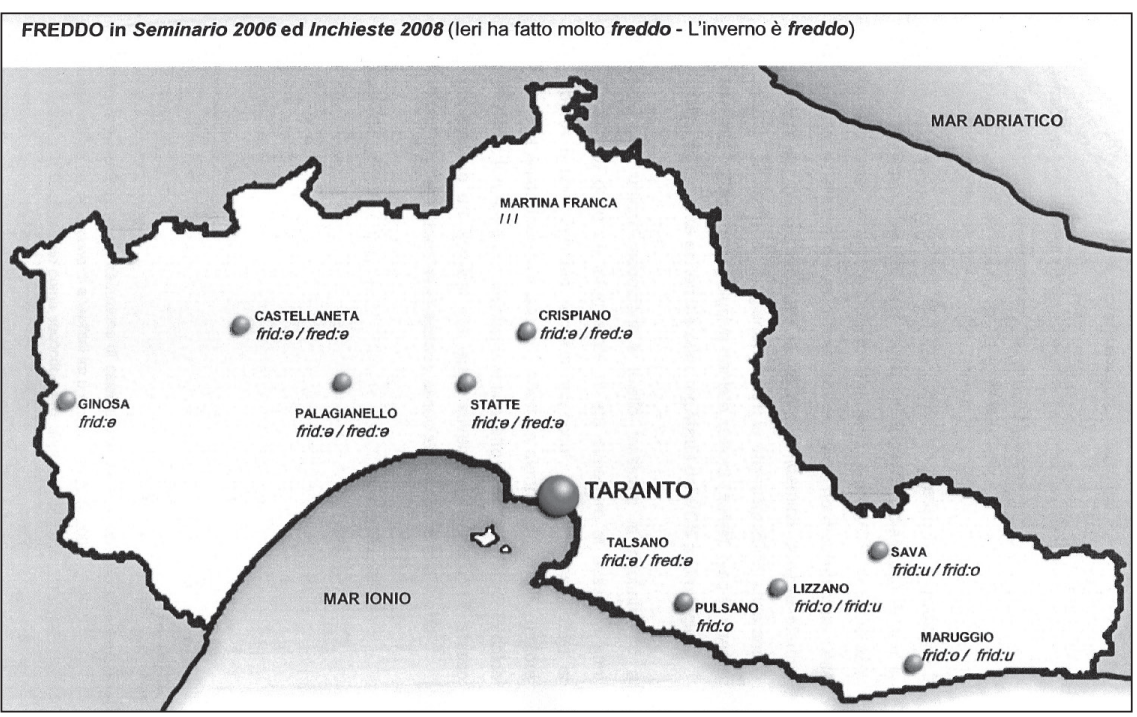

Dans Inchieste 2008, pour le terme caldo 'chaud', sur un échantillon de 100 personnes, 97 informateurs produisent le type lexical italien avec des variantes phonétiques dialectales. Encore une fois, par rapport à ce qui était enregistré dans VDS, il n'y a que de petites différences au niveau phonétique : dans la zone nord-occidentale de la province le VDS indiquait la variante endémique calla (à Martina Franca, Massafra et Palagiano) alors que dans Inchieste 2008, on retrouve la variante italienne avec consonne occlusive dentale sonore (kaldə). En outre, la variante endémique còtu, mentionnée dans VDS à Grottaglie, n'est pas référencée.

Les seules exceptions au niveau lexical sont attestées à Crispiano, là où un informateur âgé de plus de 60 ans emploie le type lexical for:ok:ə 'sirocco' et à Ginosa, où l'adjectif afosa 'étouffante' (sous-entendu chaleur) est utilisé par un informateur lui aussi âgé de plus de 60 ans. Le chaud est ici associé au concept de sirocco, vent chaud en provenance du sud-est qui souffle souvent dans le Salento. La même chose est valable pour la chaleur étouffante, aussi souvent associée au concept de chaud. 


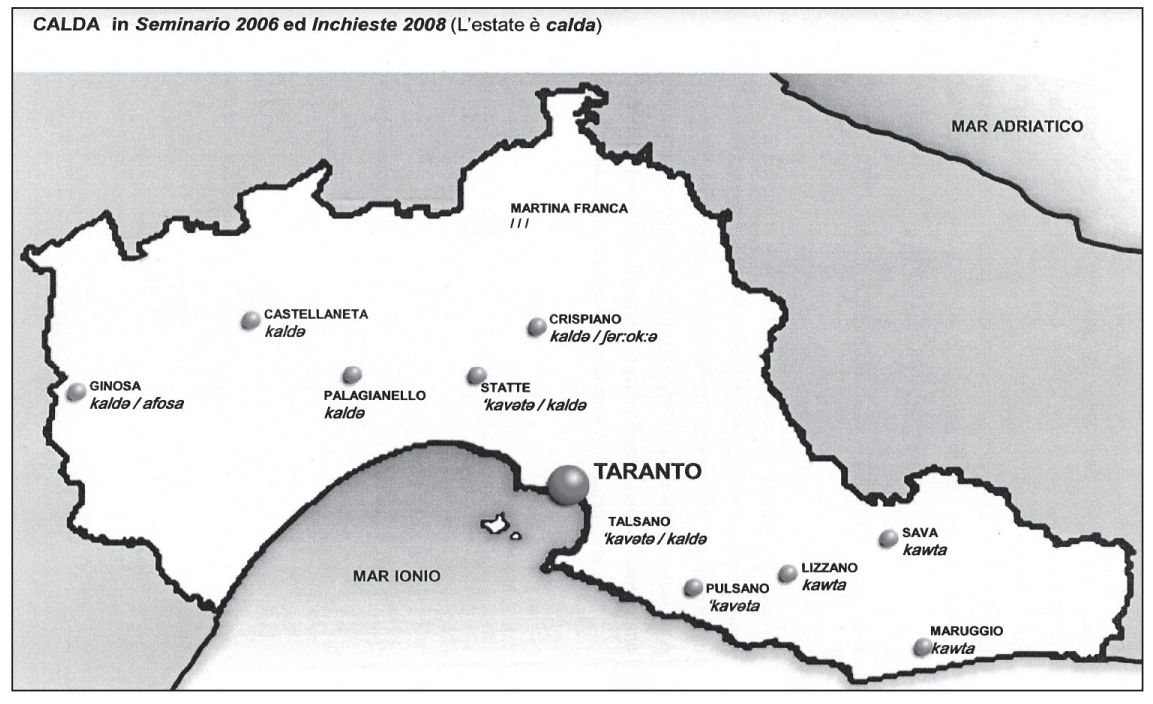

Ce qui a été remarqué pour le terme froid est aussi valable pour le vocable chaud : 5 informateurs sur 100 traduisent la phrase "L'estate è calda " par l'estate fa caldo. Ce phénomène est aussi enregistré pour les donnés du Seminario 2006, dans le point d'enquête de Talsano, pour un nombre de 3 informateurs sur 6.

Tout cela confirme l'observation de Tappolet: "Quanto più un fenomeno atmosferico si presenta come concetto generale, tanta maggiore concordanza ed uniformità mostrano le corrispondenti denominazioni dialettali» (Tappolet, 1895, p. 5). Froid et chaud sont des «météores générales », des phénomènes atmosphériques qui présentent une très grande uniformité des résultats même dans Inchieste 2008.

\section{Pleuvoir, pleuvioter}

Pleuvoir et pleuvioter sont deux cas intéressants pour montrer la différence essentielle entre les dénominations des concepts météorologiques de base et les dénominations des concepts changeants et nuancés. Pleuvoir appartient à la première catégorie, il s'agit d'un phénomène météorologique de base, tandis que pleuvioter est un concept changeant et nuancé.

D'après Lazzari, la pluie et le pleuvoir sont extrêmement importants pour l'économie mondiale et, mise à part leur quantité, il s'agit de concepts déterminés, généraux, constants et invariables : cela détermine une grande uniformité dans les expressions employées par les locuteurs pour les nommer (Lazzari, 1919, p. 8). À partir d'un aperçu des dialectes d'Italie, 
Lazzari constate que tous font dériver le vocable pleuvoir du latin vulgaire plövere (et non du latin classique pluĕre), tandis que pour le mot pluie, des trois termes du latin classique (pluvia, pluor, aqua), seul le vocable pluvia survit aux autres, qu'il soit employé de façon isolée ou accompagné par d'autres mots (Lazzari, 1919, p. 8). Il n'y pas de nouvelles créations ni pour pleuvoir ni pour pluie. Quant à pleuvoir, on remarque seulement l'emploi d'autres conjugaisons (Lazzari, 1919, p. 12). Or, de même que pour froid et chaud, l'observation de Tappolet citée auparavant est ici valable : la pluie et le pleuvoir, étant des «météores générales», présentent une très grande uniformité des dénominations.

La province de Tarente n'est pas une exception. Pendant toute la période de temps analysée, pleuvoir est toujours nommé à travers le même type lexical : 'kjovere (avec des variantes phonétiques dialectales) du latin vulgaire plövere (avec transformation de $p l$ - en $k j$ - typique des dialectes de l'Italie méridionale).

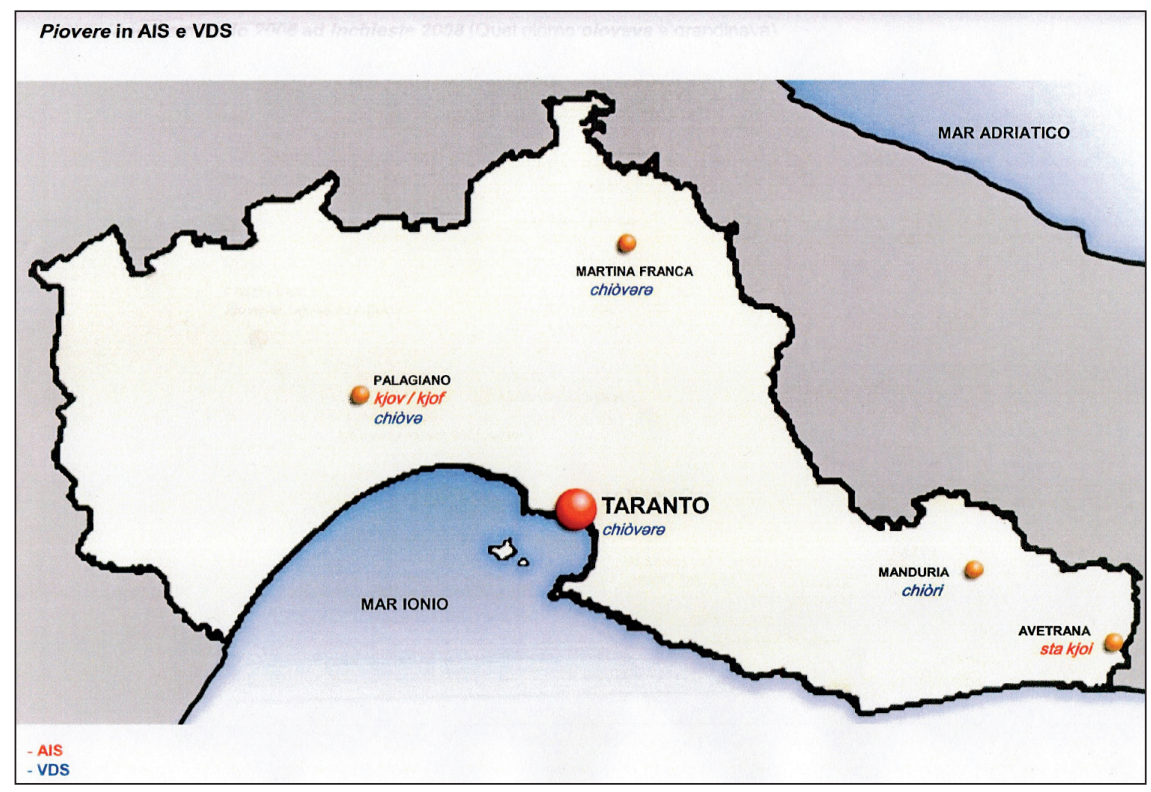

Dans Inchieste 2008 aussi, le type lexical enregistré est 'kjovere pour 98 occurrences sur 100 (avec des variantes phonétiques), à partir du latin vulgaire plövere. La phrase proposée était «Quel giorno pioveva».

Seulement un hapax est répertorié : un jeune informateur de Statte, pour traduire pioveva 'il pleuvait', emploie la périphrase kadeva ak:wə 'il tombait de l'eau/de la pluie'. Le même phénomène s'est produit dans Seminario 2006 qui enregistre pour un informateur âgé de Talsano ste fafevo tayt ak:wa 'il tombait beaucoup d'eau/de pluie'. 


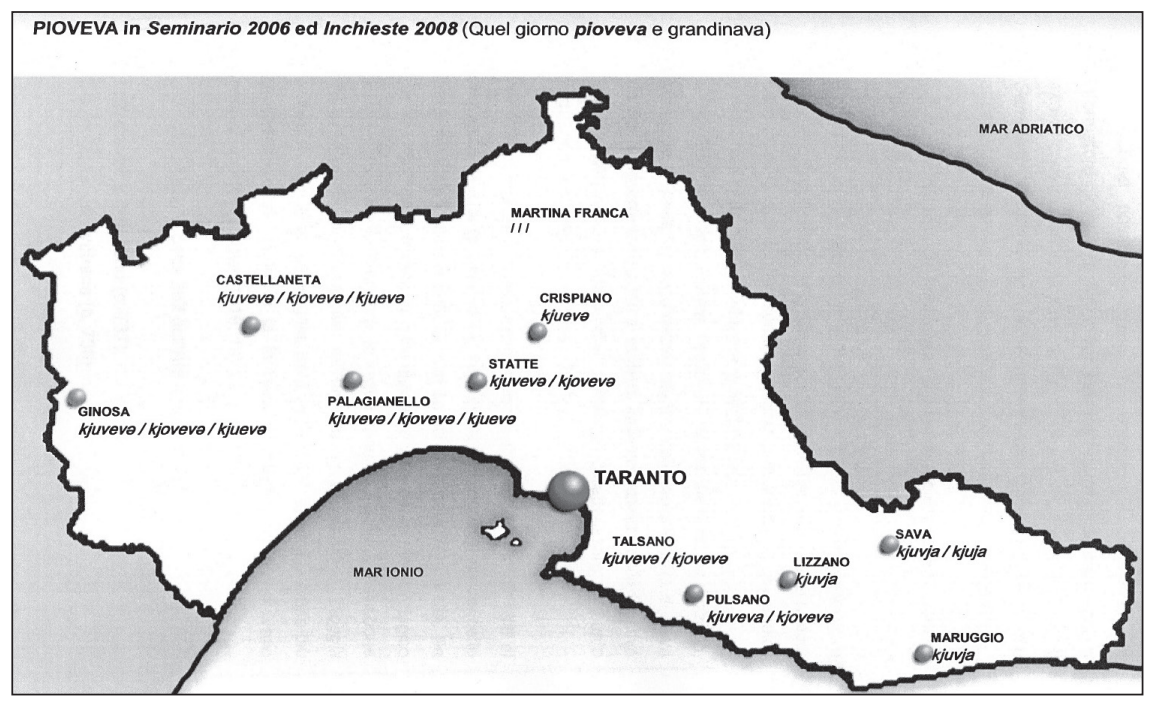

Concernant les résultats de pleuvioter, la situation est très différente. En effet, d'après Lazzari, la pluie est un concept déterminé et général mise à part sa quantité. En revanche, si ce dernier élément est pris en compte, la pluie devient un phénomène météorologique très variable. En général, les langues n'appliquent qu'une seule distinction entre bruine d'un côté et averse de l'autre. Cependant, en réalité, la pluie présente plus de changements et de nuances. L'imagination populaire est donc amenée à créer des expressions diverses (Lazzari, 1919, p. 16).

Pour pleuvioter, le type lexical ntsìddikare dans différentes réalisations est attesté dans AIS à Palagiano et Avetrana. En revanche, dans VDS, pour la seule province de Tarente, on enregistre :

1) le type lexical nziddicare à Tarente, Martina Franca, Avetrana et Manduria (à partir de nzidḍ 'goutte', terme probablement dérivé - selon Rohlfs - du latin uncilla 'petite once'2) dans différentes variantes;

2) piulisciari à Sava (avec la signification littérale 'pleuvoir à petits poils');

3) priulasciá à Martina Franca, pruvulèscia à San Giorgio (de pròule 'poudre');

4) ranisciare (qui signifie 'pleuvioter à graines rares et menues');

2. Probablement du lat. STILLA (cf. REW 8258). 
5) ріu рiu à Manduria à l'intérieur de la locution l'acqua píu píu 'la bruine, la pluie toute fine'.

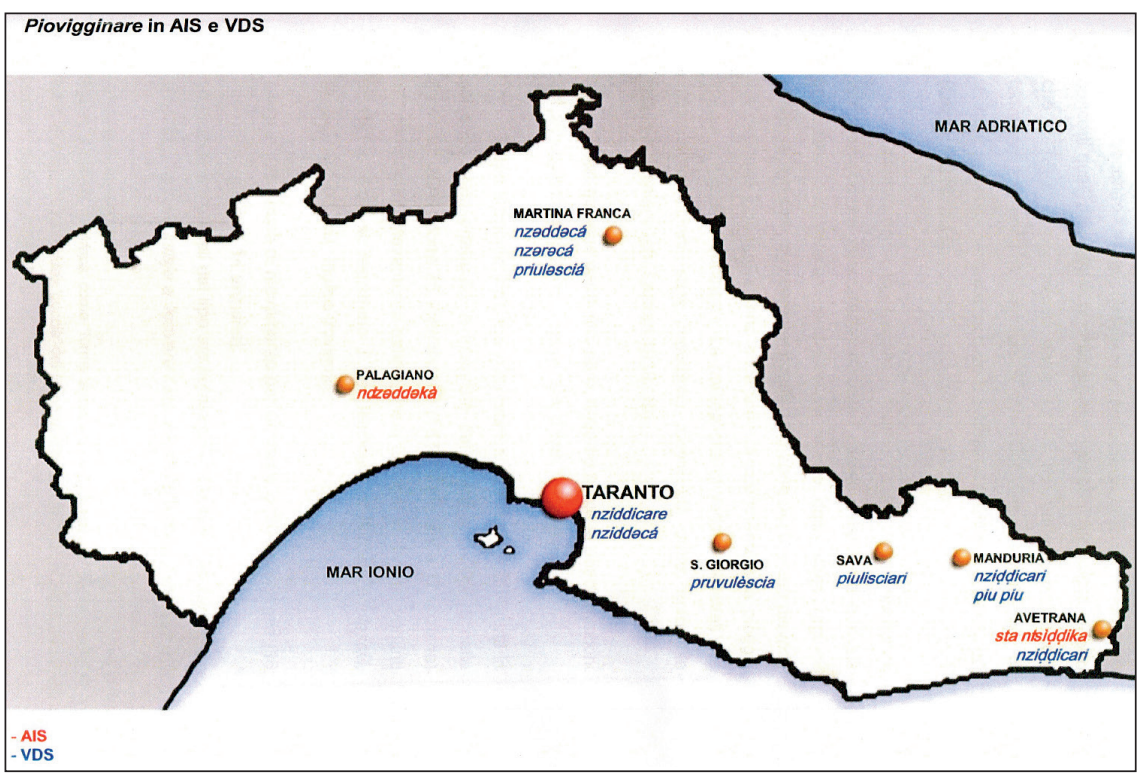

Par conséquent, afin d'exprimer le concept de pleuvioter, le locuteur s'appuie sur sa créativité, qui s'inspire des images de la vie quotidienne, pour créer des similitudes. Cela expliquerait pourquoi l'on retrouve différents termes icastiques, des termes qui décrivent la réalité à travers des images, d'une évidence représentative (Sobrero et Miglietta, 2006, p. 148).

Selon Lazzari (1919), le fait de donner libre cours à l'imagination populaire sur des concepts tels que bruine ou pleuvioter serait à attribuer au fait que «il bisogno più sentito dagli uomini nel parlare, anche dei fenomeni più comuni, sia quello, per così dire, di realizzare, di rendere sensibile nella parola tutte le impressioni nella loro pienezza» (p. 16). De plus, par le biais de Lazzari, on constate comment une telle richesse terminologique se retrouve dans tous les dialectes d'Italie, mais n'est pas due à des conditions climatiques différentes dans les différentes régions. Évidemment, le long de la péninsule, il existe des zones plus ou moins pluvieuses, mais partout les locuteurs ont l'occasion d'observer des pluies faibles et des pluies abondantes. La multiplicité des variantes dialectales identifiées sera donc à attribuer, comme déjà mentionné, à la seule imagination populaire (ibid.).

Toutefois, après quelques décennies, on remarque que les dénominations de pluvioter, en général, semblent moins imaginatives. Le type lexical 
indigène $\eta^{\prime}$ tsid:ika est attesté pour tous les points où les entretiens du Seminario 2006 ont été réalisés. Dans Inchieste 2008, il apparait 35 fois sur 100 (source : 18 informateurs jeunes et 17 âgés) pour tous les points d'enquête sauf pour Ginosa. Il n'y a pas d'occurrence des types lexicaux piulisciari, priulasciá, ranisciare et piu piu. De plus, il est intéressant d'observer que, pour un grand nombre d'occurrences, le locuteur emploie de véritables hypotraductions : pour 30 occurrences sur 100, pluvioter est traduit tout simplement par pleuvoir. Le phénomène est enregistré pour tous les points d'enquête, il est plus ou moins distribué de façon équitable parmi les informateurs jeunes et les informateurs âgés.

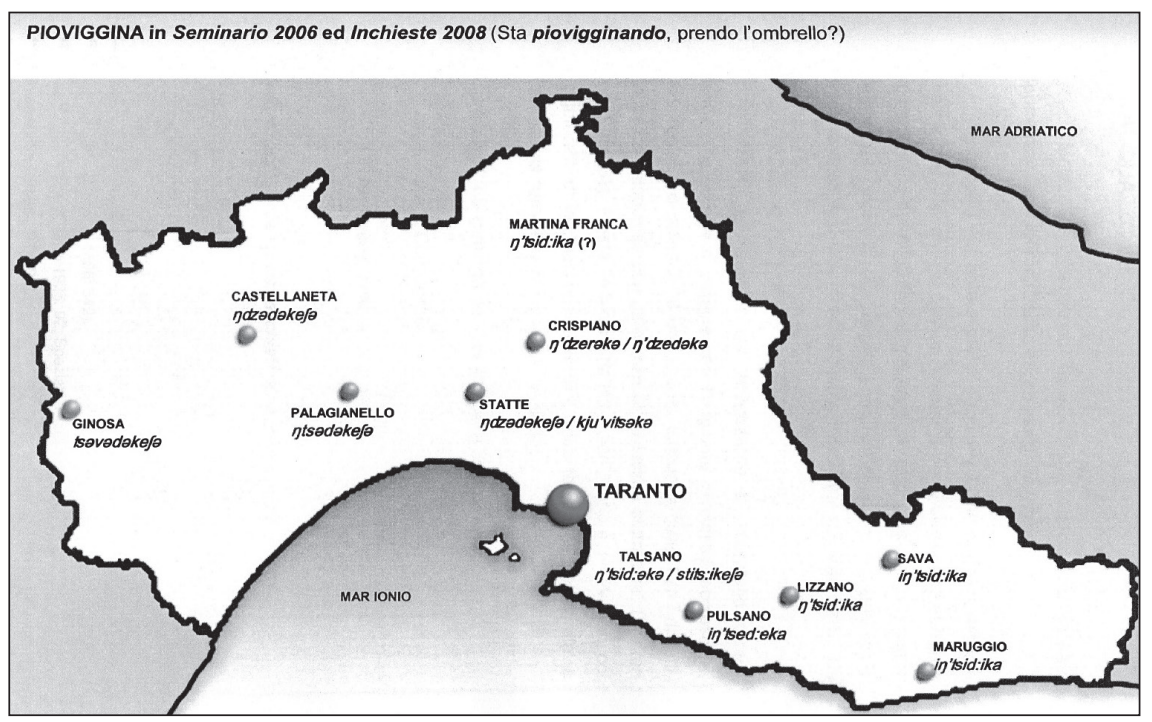

Dans 11 occurrences (relevées pour presque tous les points d'enquête, à la fois pour des informateurs jeunes et âgés), on enregistre des périphrases d'imminence qui communiquent l'idée qu'il est en train de commencer à pleuvoir, mais sans transmettre le concept de pleuvioter. La fonction du morphème -iggina modifie la signification de base (pleuvoir) et communique l'idée d'une petite bruine continue, l'idée étant perçue par les informateurs. Le parleur comprend inconsciemment que ce concept implique un profil aspectuel de durée, mais en traduisant il se perd et rejoint l'aspect inchoatif à la place de l'aspect duratif (Sobrero et Miglietta, 2006, p. 149). À cet égard, il est emblématique de citer la périphrase enregistrée auprès de Castellaneta pour un informateur âgé qui traduit directement il est en train de pleuvioter par sa stav:itfina 'il est en train de s'approcher' où le concept de pleuvioter est complètement omis et seule l'approche de quelque chose est communiquée. 
Dans les autres cas, enfin, le parleur cherche à remplacer certains types lexicaux disparus par des expressions syntagmatiques telles que kjove ap:ena ap:ena 'il pleut juste un petit peu' / kjova pik:a pik:a 'il pleut un petit peut'. Au contraire, dans d'autres cas, des simples calques de l'italien sont fournis (pjo'vidz:ina 'il pleuviote' / sta pjovidz:inaydo 'il est en train de pleuvioter') : Castiglione (2004) fait remarquer que la traductioncalque est sans doute «quella più aproblematica e applicabile al minimo delle competenze» (p. 63), également pour l'échantillon des informateurs jeunes et pour les sources âgées.

L'analyse des données révèle, par conséquent, que le système est aujourd'hui instable. Il s'agit d'une phase de transition : le parleur possède des compétences morphosémantiques qui apparaissent réduites et sa créativité semble très atténuée, dominée par la puissance de l'innovation qui l'anime et le dirige vers les formes propres à l'italien.

\section{Averse}

La plupart des observations faites pour le météoronyme pleuvioter pourraient également être considérées valables pour le mot averse.

Lazzari attire l'attention sur le fait qu'il n'y a pas de traces du terme du latin classique imber - imbris dans aucun des dialectes d'Italie. Il s'agit donc de «nuove creazioni, la cui origine, per la maggior parte, può essere quella tendenza naturale al popolo di esprimere le proprie impressioni in forma alquanto iperbolica» (Lazzari, 1919, p. 32-33).

Dans l'Italie des dialectes, pour le vocable averse, il y a différents dérivés des mots acqua 'eau' et pioggia 'pluie' auxquels s'ajoutent différents suffixes augmentatifs, péjoratifs ou collectifs «sì che le voci, comuni a quasi tutti i dialetti, appaiono sinonimie con differenze di lievissime sfumature» (ibid.). En outre, les termes latin temperies 'orage' et tempestas 'tempête' ont survécu avec la signification d' 'averse' dans certains dialectes.

Dans AIS on constate nn akwa muta à Carovigno. Le VDS, au contraire, signale acquarone et sfrušciácchiə/sprušciácchiz à Martina Franca.

Dans les données collectées pour le Seminario 2006 ces types lexicaux n'apparaissent plus et, au bout de plus d'un demi-siècle, le résultat courant s'avère être le type lexical italien acquazzone 'averse' (caractérisé par des variantes phonétiques de la zone).

Dans Inchieste 2008, le terme compte 69 occurrences sur 100 et il est attesté dans tous les points d'enquête. Cependant, la situation est plus nuancée par rapport à ce que l'on a enregistré pour les autres météoronymes : on constate une prolifération des variantes plus accentuée que dans les autres cas. 


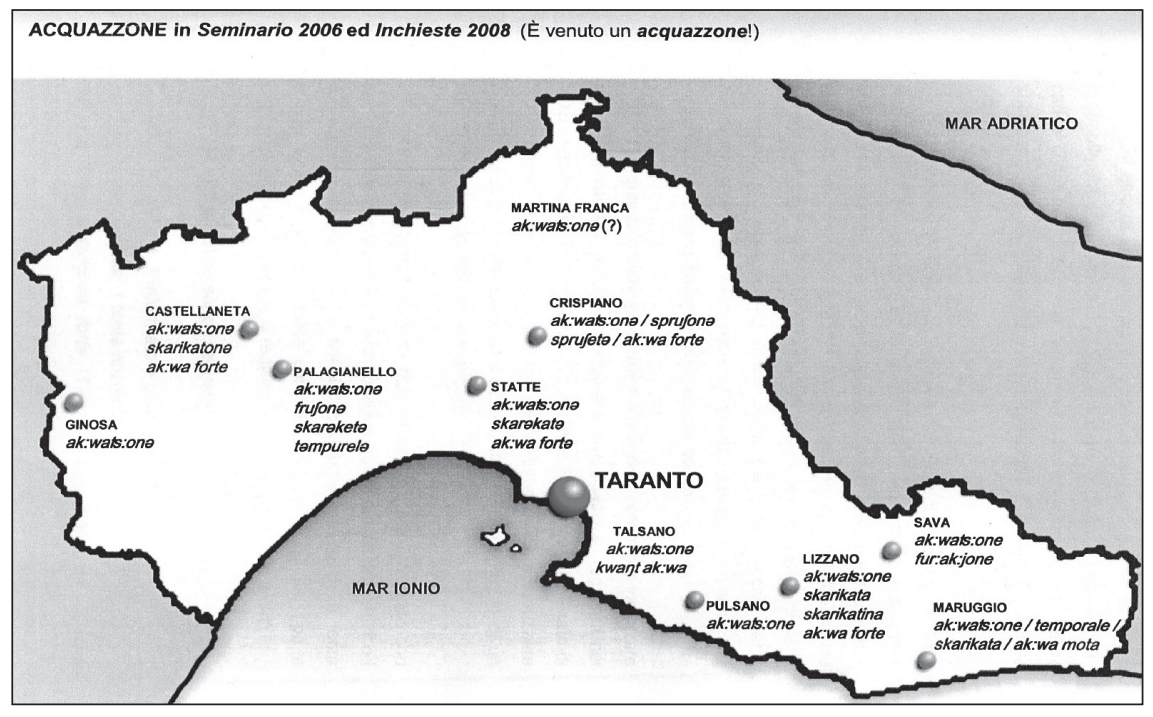

Par conséquent, on enregistre :

1) les résultats du type skarikata/skarikatone/skarikatina qui dérivent probablement du verbe scaricare 'décharger' ('ôter la charge, libérer la charge', cf. Zanichelli, 2005). Évidemment, pour les locuteurs c'est comme si pendant une averse toute l'eau du ciel se déchargeait sur terre, le ciel étant chargé avant l'arrivée de l'eau;

2) les traductions du type ak:wa forte/ak:wa mota/ak:wa qui expliquent le concept d'averse à travers l'association de l'hypéronyme acqua 'eau' et d'adjectifs qui communiquent un sentiment d'abondance et qui permettent de rapprocher la signification du terme à celle d'averse. Auparavant dans AIS, pour averse il y avait la forme nn akwa muta à Carovigno. Le phénomène est aussi bien enregistré pour les données collectées lors du Seminario 2006 : un informateur de plus de 60 ans de Talsano pour averse fournit la réponse kwayt $a k: w a$ 'combien d'eau';

3) les résultats du type sprufona/sprufeta : on pourrait supposer qu'il s'agit de variantes des types sprušciata et sprušciácchio, enregistrés dans VDS, respectivement à Squinzano et Martina Franca, avec la signification d'averse. Ces résultats pourraient dériver du verbe sprušciare 'asperger du liquide' et/ou du substantif sprušciata 'jet ou éclaboussure d'un liquide’ répertoriés par Rohlfs à Mesagne. 
Par ailleurs, il est à noter que le terme averse est fréquemment traduit par des périphrases composées par l'association du verbe pleuvoir et d'adjectifs ou adverbes qui communiquent un sentiment d'abondance et de force (tels que fort, beaucoup) ou, même, par des locutions adverbiales telles que (il pleut) à verse, à torrents, (il pleut) fort. Dans un seul cas on enregistre le verbe pleuvoir tout court. Dans ces cas-là, les informateurs paraphrasent l'expression en obtenant des traductions sémantiques (Castiglione, 2004, p. 55-63) à travers l'emploi de stratégies de détournement qui visent à l'omission de l'élément problématique (ibid., p. 123). Cependant, le risque est de tomber dans des hypotraductions comme c'est le cas pour la phrase «È venuto un acquazzone " traduite par ste kjova 'il est en train de pleuvoir' (Crispiano, source : informateur jeune) et $s$ a $m: i s a$ a kjovo 'il s'est mis à pleuvoir' (Castellaneta, source : informateur âgé) : dans ces occurrences on communique seulement sur le fait qu'il pleut, ou qu'il va commencer à pleuvoir, et non pas sur l'arrivée d'une averse. Un informateur âgé de Pulsano traduit la phrase «Une averse est arrivée! » par l'expression tfe ak:wa se'ne venuta, kome ste kjove!! 'combien d'eau est arrivée, comme il pleut!!'. Dans le cas en question, la tonie exclamative marquée comble le vide lexical.

\section{Grêle, grêler}

En comparaison avec ce qui est attesté dans AIS, ALI et VDS pour grêle/ grêler, les données collectées pour le Seminario 2006 et pour les Inchieste 2008 dans la province de Tarente témoignent d'un abandon progressif des variantes phonétiques les plus archaïques. Le type lexical italien, présent dans tous les points d'enquête, est enregistré avec différents niveaux de dialectisation. Sur le plan phonétique, il est question d'une forte poussée novatrice et l'innovation est d'autant plus forte dans les centres les plus proches de la ville (Talsano et Statte, où les variantes phonétiques endémiques ne sont pas enregistrées) car, d'après Castiglione (2004),

la contemporanea mancanza di un ambiente socio-culturale che mantenga il dialetto e di un ambiente cittadino che preservi ambiti d'uso dialettale, produce uno sfarinamento delle competenze, lessicali, morfosintattiche $[\ldots]$ e fonetiche [...], soprattutto presso i giovani (p. 27). 

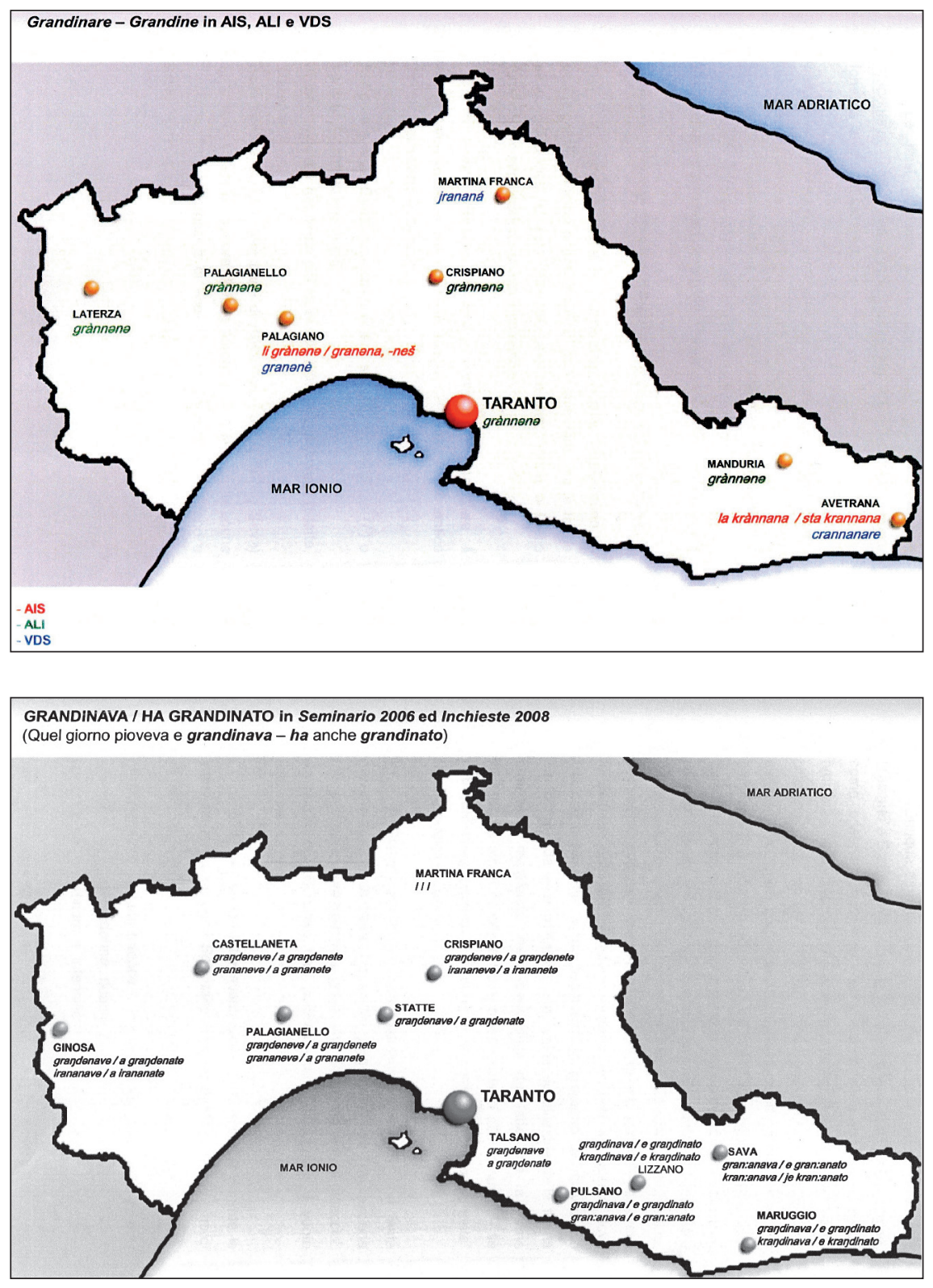

On peut s'attendre à ce que cette innovation conduise au prompt remplacement des traits phonétiques archaïques, dont la plupart sont aujourd'hui attribués à des sources d'informateurs âgés et dans les points d'enquête les plus éloignés de la ville. De plus, le remplacement pourrait être irréversible : c'est possible que, sur le plan phonétique, il se produise le même 
phénomène qui a eu lieu dans le Salento où Sobrero et Miglietta (2006) ont observé un processus d'innovation

tanto forte da aver portato a una rapida sostituzione della forma endemica, metaforica, resa irreversibile dal fatto che la forma sostituita è ormai percepita come troppo interna alla comunità - se non addirittura criptica e dunque è connotata negativamente (p. 151).

À Statte, une traduction différente a été notée : un jeune informateur traduit la phrase «Ha anche grandinato $"$ par l'expression métonymique a p:ura menata u gjat:a 'il est même tombé de la glace'. Dans ce cas aussi, une périphrase vient combler le vide lexical.

Enfin, certains résultats enregistrés au cours des entretiens effectués pour le Seminario 2006 dans le point d'enquête de Talsano semblent intéressants. Un informateur âgé traduit «...e grandinava» par ma'do kwanta graydənə ka fat:ə 'Sainte Vierge, combien de grêle qu'il a fait', où le concept de grêler est renforcé par l'interjection Sainte Vierge et par l'intégration du texte à travers l'expansion du même (Romanello, 1995, p. 127). Encore une fois, un informateur de plus de 60 ans traduit la même phrase par madon:a te mala timpa 'Sainte Vierge, quel mauvais temps', car il identifie le phénomène du grêler avec le mauvais temps, mais cela produit une hypotraduction. Pour finir, un autre informateur âgé traduit la phrase «ha anche grandinato" par a p:urə kjuvutz fortz 'il a aussi plu fort' et l'on pourrait supposer que la source, ayant oublié le terme dialectal qui exprime les mots grêle/grêler, interprète le concept en intensifiant le verbe pleuvoir à travers la juxtaposition de l'adverbe fort, comme si la grêle était une forte pluie.

\section{Neiger, Neige}

Lazzari note que pour le terme neige il y avait en latin classique deux expressions : nix et ninguis, avec une plus grande diffusion de la première qui survit dans presque tous les dialectes italiens et romans (Lazzari, 1919, p. 45). Cependant, pour le verbe neiger, en règle générale, les dialectes d'Italie reprennent les mots préromans nivare, nivicare et non pas les vocables du latin classique (ibid., p. 50). La situation est confirmée dans AIS et VDS.

Dans Inchieste 2008 pour la phrase «Quest'inverno ha nevicato» dans tous les points d'enquête les résultats s'alternent entre il a neigé et la forme substantivée il a fait la neige (avec les variantes phonétiques habituelles). Même dans la traduction en dialecte, comme en italien, neiger est conjugué à la fois avec l'auxiliaire être et avoir, mais les occurrences avec l'auxi- 
liaire être sont enregistrées exclusivement dans les points d'enquête au sud-est de Tarente, sauf pour Talsano où l'on retrouve la construction avec le verbe avoir.
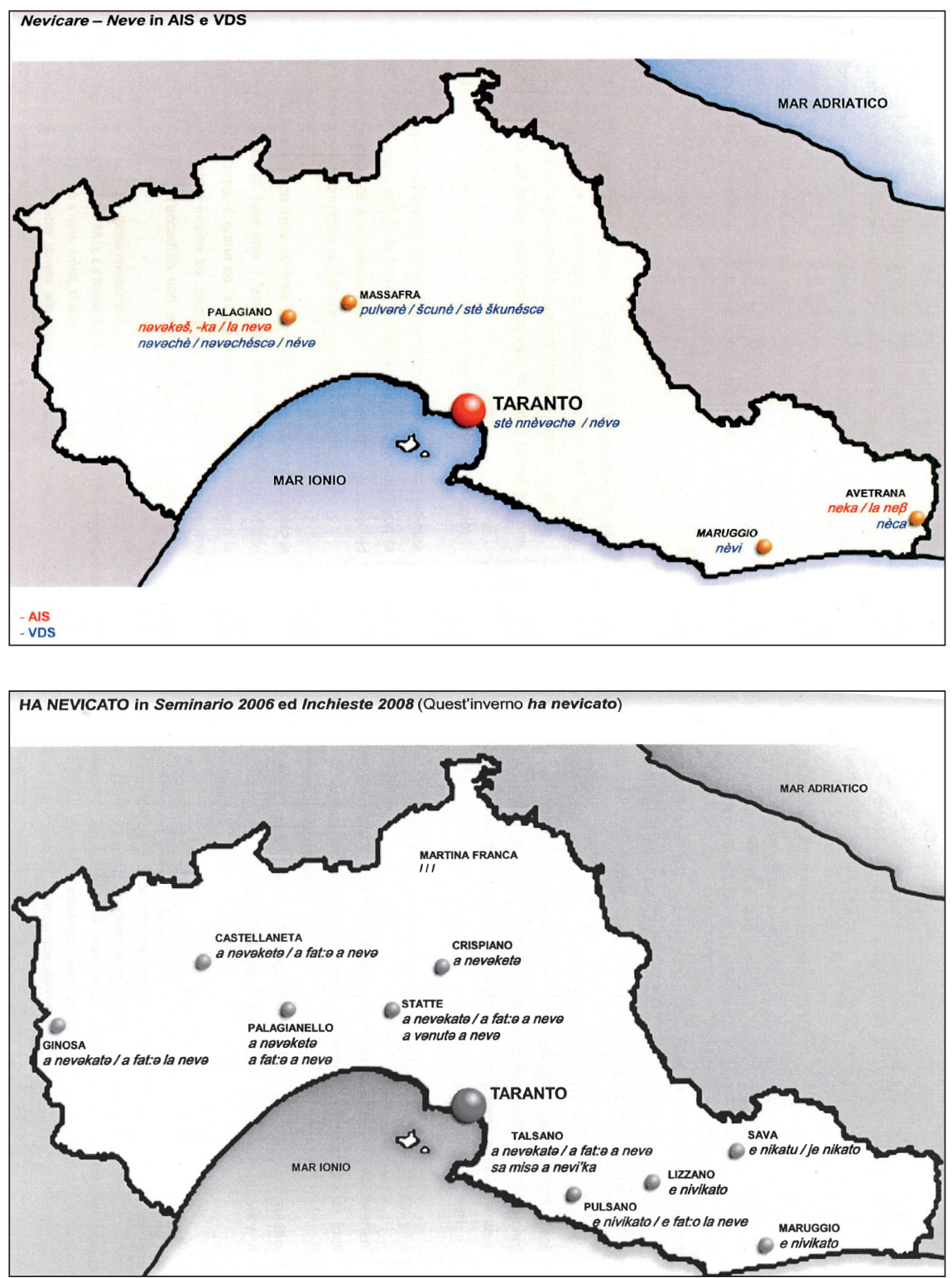

Une occurrence très intéressante est donnée par un informateur jeune de Talsano qui traduit la phrase «Cet hiver il a neigé » par la périphrase 
aspectuelle $s$ a misa a nevi'ka 'il s'est mis à neiger'. Il interprète donc à travers un filtre aspectuel une catégorie temporelle.

L'emploi de périphrases amo vifto la neve et amo provato la neve est aussi typique de cette région, où le phénomène du neiger n'est pas du tout récurrent. C'est comme si l'informateur était surpris de voir la neige : cela explique pourquoi il a tendance à utiliser des verbes tels que faire l'essai de et voir ou à souligner l'événement par des périphrases aspectuelles (voir ci-dessus). Enfin, l'étonnement de l'informateur face au phénomène est remarquable également pour un entretien effectué à Talsano pour le Seminario 2006 : une source de plus de 60 ans traduit la phrase «Cet hiver il a neigé » par la périphrase kwanta nevə ka fat:ə 'combien de neige qu'il a fait', c'est la tonie particulièrement marquée qui communique le sentiment de merveille pour ce résultat.

Probablement la rareté du phénomène est la cause de la grande uniformité des résultats. Effectivement, Lazzari note que dans tous les dialectes italiens il y a peu de nouvelles créations et qu'elles sont limitées à ces zones géographiques où il neige plus souvent (Lazzari, 1919, p. 45); la rareté de la neige pourrait aussi expliquer «la confusione, frequente nelle regioni meridionali, di chiamare neve il 'ghiaccio'e viceversa» (ibid., p. 51), constatation faite également par De Vincentiis qui indique chiatro en tant que terme qui désigne la glace mais aussi la neige glacée (De Vincentiis, 1872, p. 63).

Une autre hypothèse serait d'attribuer la grande uniformité des résultats enregistrés pour neige et neiger à leur nature de phénomènes météorologiques ou faits percepteurs de base. Cela viendrait confirmer ultérieurement l'observation de Tappolet citée précédemment lors de l'analyse de froid et chaud, pluie et pleuvoir (Tappolet, 1895, p. 5).

\section{Tonnerre}

Dans AIS, ALI et VDS le météoronyme est traduit par le seul type lexical tronu/truenu (du latin tonitrus) et les résultats fournis dans Seminario 2006 et Inchieste 2008 produisent des données en parfaite continuité avec les résultats de la bibliographie.

La seule différence significative est représentée par le résultat d'un informateur de plus de 60 ans de Maruggio qui traduit tonnerre par orage, par contiguiité sémantique. Presque le même phénomène est enregistré pour une source de plus de 60 ans de Talsano (interviewée lors du Seminario 2006). L'informateur, probablement dépourvu d'un correspondant dialectal pour tonnerre, propose le résultat so le rifles:a du temporala 'ce sont les reflets de l'orage'. 

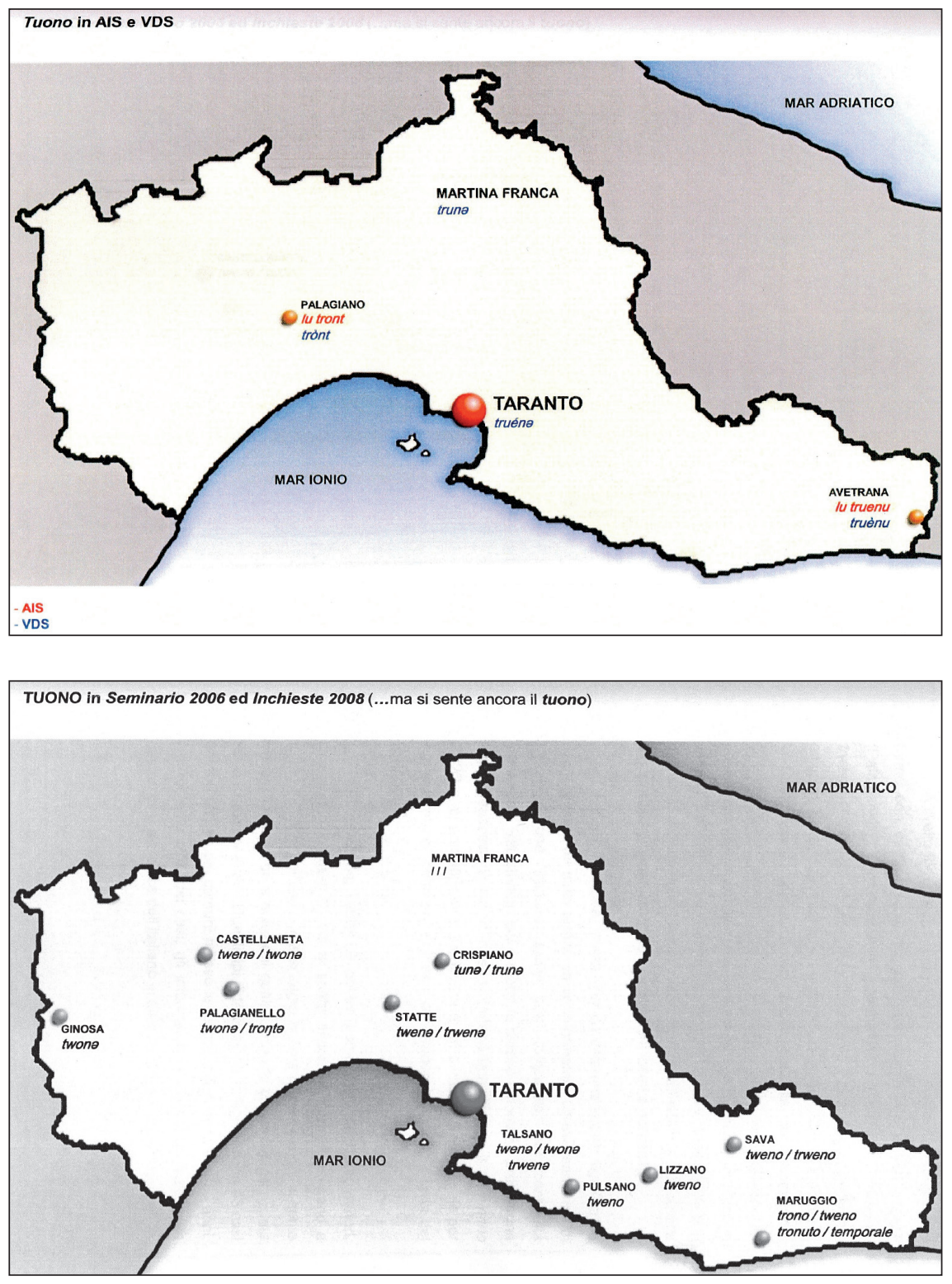

En outre, il est intéressant de remarquer comment la plupart des informateurs, en traduisant la phrase «... ma si sente ancora il tuono», produisent des formes infinitives telles que ... ma si seyte ajkora trunare '... mais on entend encore tonner' ou des formes comme ... ma ste ytrona a kkora '... mais il est encore en train de tonner'. Ces types de traduction sont répertoriés pour 43 cas sur 100 et ils sont plus répandus chez les 
sources ayant un âge supérieur à 60 ans. Pour $57 \%$ des cas qui restent, les informateurs se limitent à calquer la construction syntaxique de l'italien en dialecte, sans modifications.

\section{Foudre}

Dans AIS, pour la province de Tarente, on enregistre lu fulmənə à Palagiano et lu lampu à Avetrana. Dans VDS le terme 'foudre' renvoie à 'éclair', nous retrouvons lampu à Avetrana, sajèttz à Martina Franca avec la signification de 'foudre, éclair' et le type tirlampu dans différentes variantes phonétiques à Sava et Tarente.

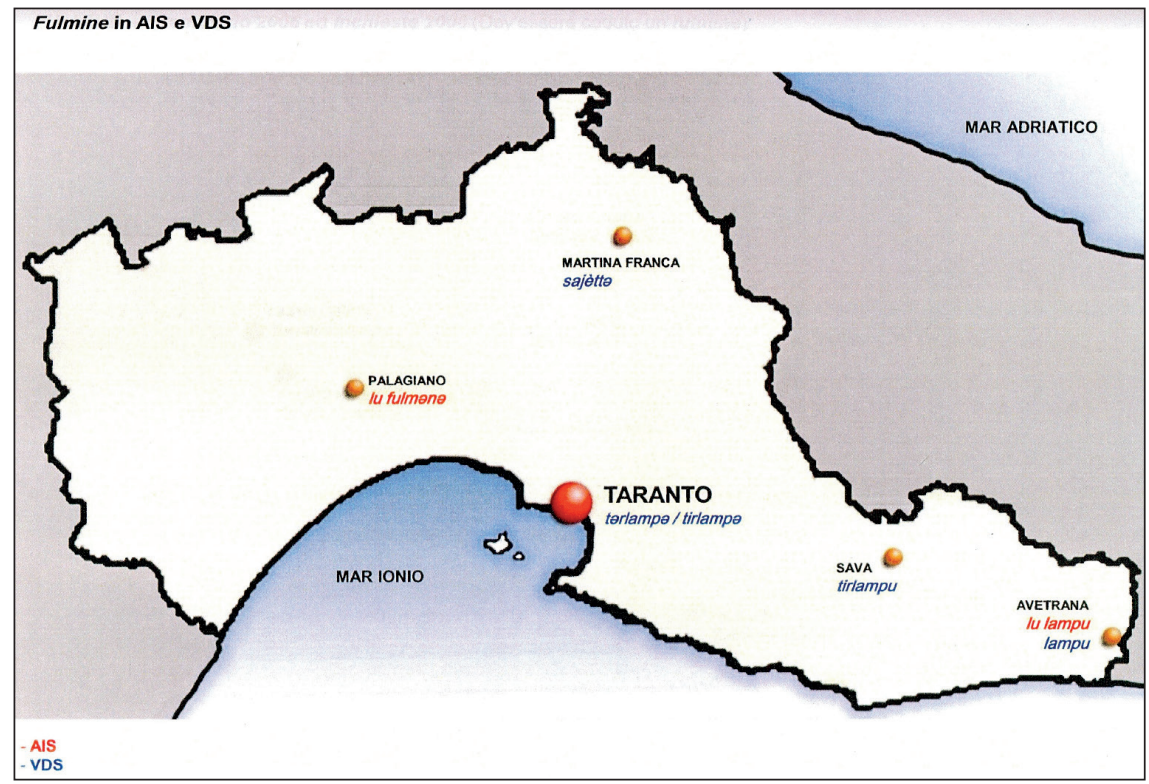

À partir de l'analyse des données collectées dans la province de Tarente par le biais du Seminario 2006 et des Inchieste 2008, un fort processus d'italianisation est noté. Sur 100 occurrences, dans Inchieste 2008 le vocable est traduit 89 fois par fulmana/fulmine 'foudre' (type lexical italien attesté dans tous les points d'enquête avec les variantes phonétiques attendues).

Pour 6 occurrences sur 100, foudre est traduit par le type lampa/lampo 'éclair' (à Castellaneta, Lizzano, Sava et Maruggio par 2 informateurs jeunes et 4 âgés). La traduction de foudre par le terme éclair est ancienne (déjà enregistrée dans AIS et VDS), cela s'explique par le fait que la foudre et l'éclair sont deux phénomènes strictement liés. Cependant, nous remarquons que le type tirlampu, certifié dans VDS à Sava et Tarente et attesté 
aussi dans les œuvres de De Vincentiis et Gigante, n'est pas enregistré dans Seminario 2006 et Inchieste 2008.

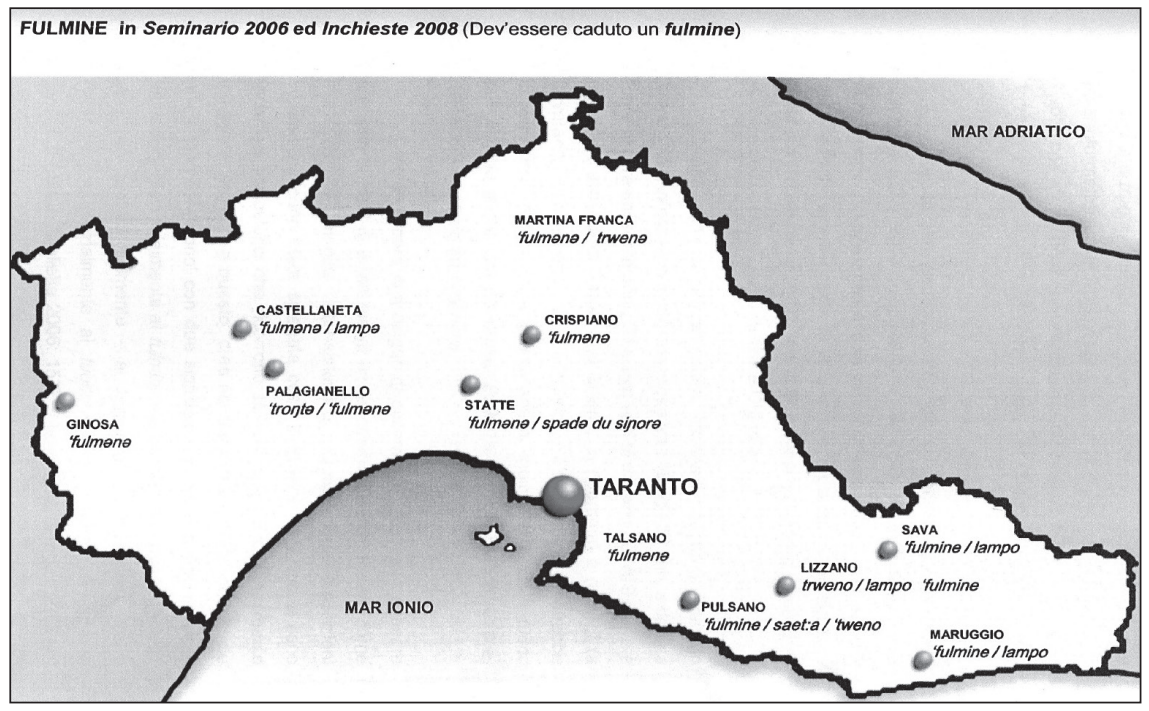

En outre, foudre est traduit 3 fois par le mot tonnerre par des informateurs d'un âge compris entre 18 et 60 ans. Les mêmes résultats apparaissent dans AIS (informateur agé de Salve, dans le sud du Salento) et dans ALI (à Leverano, Melpignano et Cisternino) : le phénomène pourrait donc être considéré relativement ancien. De plus, on enregistre tonnerre pour foudre aussi dans Seminario 2006 (à Martina Franca, Lecce, Taviano, Casarano et Andrano).

Compte tenu du nombre considérable d'occurrences, on peut imaginer qu'il ne s'agit pas d'erreurs mais d'un phénomène systémique, ou plus précisément inter-systémique. Ces superpositions sémantiques pourraient s'expliquer ainsi : la foudre et le tonnerre sont des phénomènes atmosphériques qui se produisent toujours quasi simultanément pendant les orages. Cela

crea nel parlante un'unità concettuale unica 'lampo + tuono' con due significanti in parte fungibili: lampo rimanda preferenzialmente al fulmine ma può anche rimandare - sia pure non preferenzialmente - al tuono, e reciprocamente tuono rimanda preferenzialmente al tuono e non preferenzialmente al lampo (Sobrero et Miglietta, 2006, p. 157).

Le phénomène est enregistré déjà en 1925 à Salve (AIS) mais, comme nous avons pu le constater, encore aujourd'hui il est attesté dans plusieurs points d'enquête du Salento, même chez les jeunes informateurs. 
D'après Sobrero et Miglietta (2006), le phénomène

in ipotesi si può dunque addebitare all'incontro-scontro con l'italiano, e si può classificare tra i fatti di vaghezza semantica, ovvero di plurifunzionalità lessicale, determinati non da divergenze fra i due sistemi in contatto - in questo caso infatti i tipi lessicali, sensi e significati sono perfettamente paralleli - ma dal fatto stesso che uno dei due sistemi si sia indebolito e l'altro si sia rafforzato. Se quest'ipotesi è vera, essa dimostra che vaghezza semantica, proliferazione di varianti e polisemia possono essere originate dal contatto linguistico anche indipendentemente dall'esistenza di conflitti specificatamente semantici (aree di significato non sovrapponibili, introduzione di significati e di sensi nuovi e di neologismi, abbandono di referenti e di forme desuete, ecc.) (p. 157).

Le météoronyme foudre est aussi traduit, pour une seule occurrence, par saet:a 'éclair' (Pulsano, informateur jeune), cette traduction peut être considérée de type archaïque : dans AIS, on enregistre le type saet:a pour foudre dans toute l'Italie méridionale, en faisant exception des extrêmes, dans VDS on trouve saet:a pour foudre dans le Salento septentrional, à Martina Franca.

Une traduction très intéressante est aussi fournie par une source jeune de Statte qui formule spada du sinora 'épée du Seigneur'. La foudre, par sa forme et sa dangerosité, est métaphoriquement comparée avec une épée et comme elle tombe du ciel, on imagine que ce soit Dieu à la lancer du plus haut des cieux. Dans ce genre de cas, nous pouvons nous retrouver face à des formes archaïques désormais disparues ou nous pouvons aussi imaginer que le parleur, en se retrouvant face à un probable vide lexical à combler, fasse appel à son expérience et à ses croyances, à travers l'invention d'un nouvel étiquetage créatif. Si la deuxième hypothèse était vraie, l'observation de Lazzari serait très adaptée :

Molto più ricco è il linguaggio del popolo, la cui mente colpita dal fenomeno osserva attentamente mentre la fantasia lavora e ravvicina una cosa con l'altra, scopre analogie, somiglianze, relazioni d'ogni genere e dà vita alle immagini, che si colorano variamente e si illuminano a vicenda: nasce in tal modo un parlare figurato, spontaneo, talvolta ingenио; nascono nиove espressioni leggiadre e ardite, di realistica evidenza ed efficacia, di dove meno si aspetterebbe. (Lazzari, 1919, p. 33) 


\section{Sécheresse}

L'item n'est pas prévu pour le questionnaire AIS. VDS et ALI enregistrent le type lexical siccita (proparoxyton) dans la partie centrale du Salento, les types russa/rùssala et sicca/siccarezza à la fois dans le Salento nordoccidental et méridional.

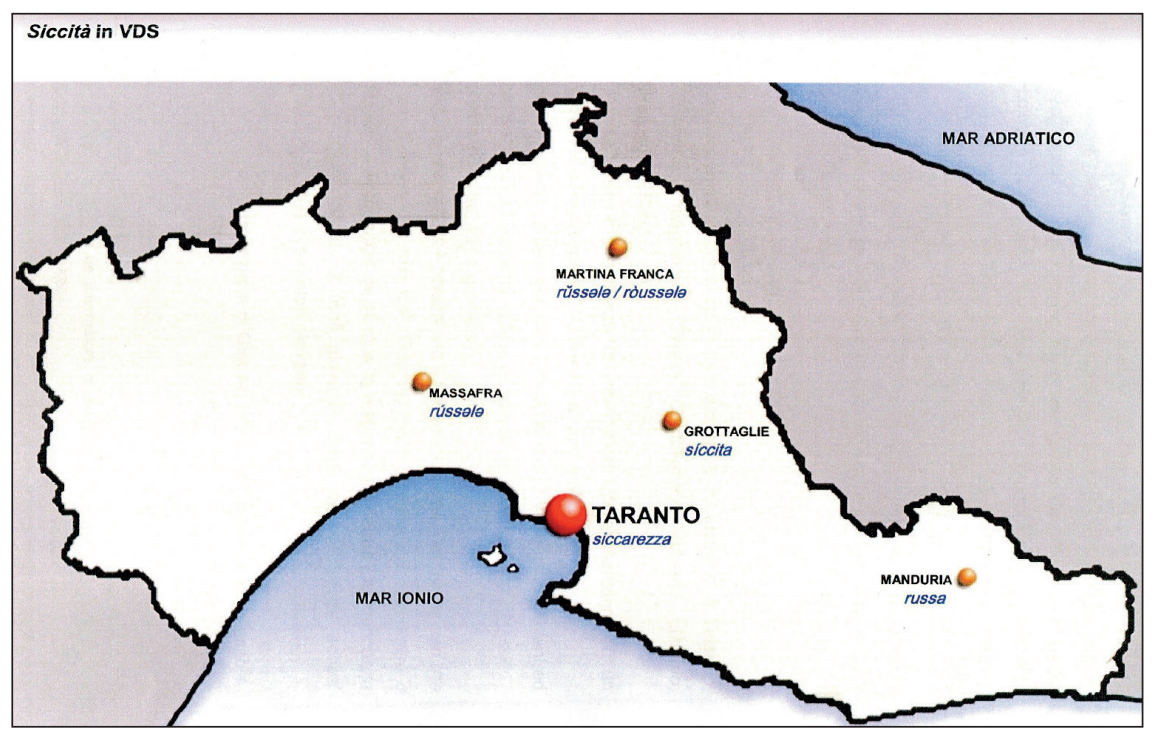

Dans Seminario 2006 le type lexical russa/rússala n'est pas enregistré, tandis que le type sicca/siccarezza apparaît à Martina Franca et Talsano. Le vide créé par le type russa est comblé par le type lexical siccita (proparoxyton) parasité par le type novateur siccità (oxyton), forme italienne attestée sur tout le territoire.

Les données des Inchieste 2008 confirment ce que l'on vient d'affirmer. Sur 100 occurrences, le type italien siccità revient 61 fois, dans tous les points d'enquête. On enregistre la disparition du type lexical russa/rússala, inversement on confirme la zone de sicca/siccarezza que l'on retrouve à Crispiano, Statte et Lizzano. Le type lexical siccita revient pour presque $30 \%$ des occurrences et il est bien attesté (on l'enregistre pour la plupart par des sources âgées dans tous les points d'enquête, sauf à Statte), mais c'est l'italien siccità qui enregistre plus du double des occurrences, il est présent dans tous les points d'enquête et le plus souvent par des sources jeunes. 


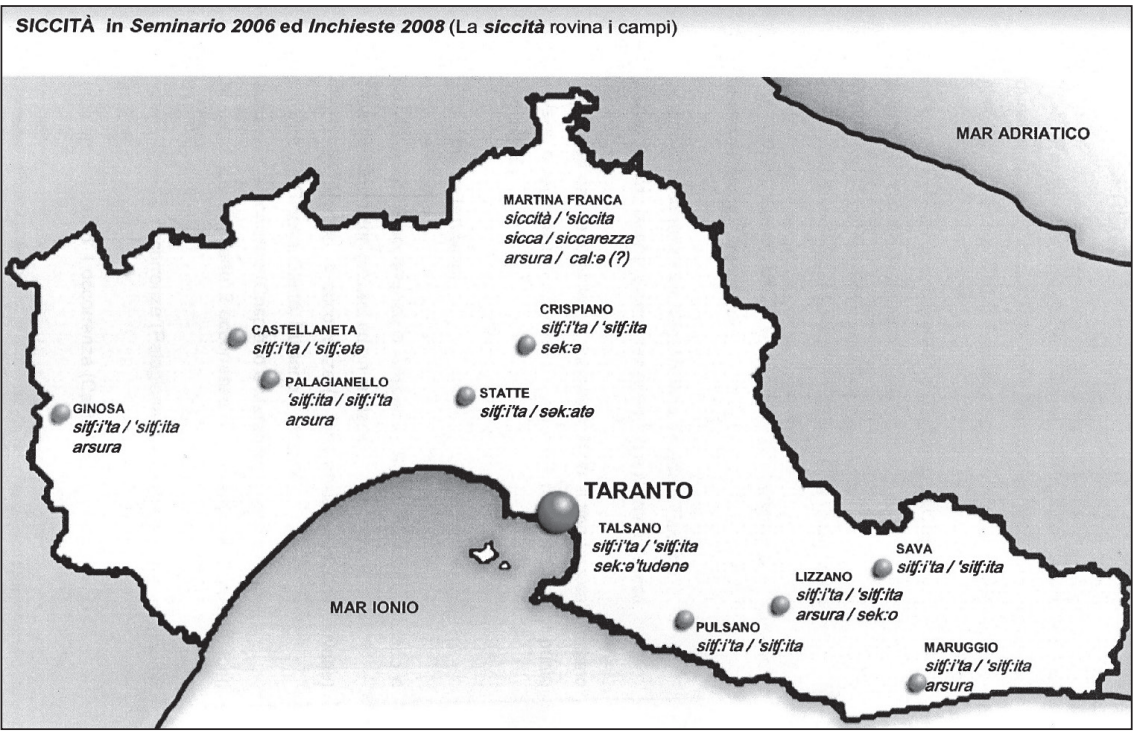

Sobrero et Miglietta (2006) observent que :

la competizione fra sìccita $e$ siccità è molto aperta e, anche in questo caso, coinvolge entrambi i sistemi linguistici in contatto: la forma tronca - più diffusa $[\ldots]$ - gode del prestigio della lingua dominante e tende a passare dall'italiano al dialetto, ma la forma sdrucciola [...] sfrutta la quasi coincidenza con la variante più prestigiosa per 'risalire' dal dialetto all' $i$ taliano regionale, e questo le conferisce una forza di risalita, nella scala delle varietà, che altre varietà dialettali non hanno $[\ldots]$ (... 160).

On constate des traductions du type arsura 'chaleur brûlante, soif ardente' et kalda 'chaud', deux métonymies : dans le premier cas, l'informateur emploie l'effet pour la cause (soif ardente < sécheresse), alors que dans le deuxième cas la source indique la cause pour l'effet (chaud $>$ sécheresse). L'utilisation de la métonymie explique aussi un probable vide lexical. Le phénomène est enregistré de même pour les résultats du Seminario 2006 : une source âgée de Martina Franca et un informateur âgé de Talsano traduisent sécheresse respectivement par arsura et ter:a arsata 'terre brûlée' (résultat obtenu dans un deuxième temps, l'intervieweuse ayant insisté); un informateur jeune de Martina Franca fournit le résultat calla 'chaud'.

Enfin, des occurrences très intéressantes sont $a k$ :wa ka non dzo ste 'l'eau

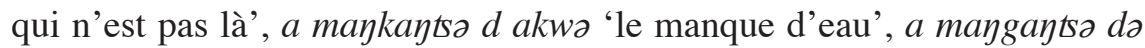
pjods:a 'le manque de pluei' et seyts ak:wa 'sans eau'. Dans ces cas-là, en se retrouvant face à un vide lexical qu'ils doivent combler, les informateurs 
(jeunes et âgés à la fois) récupèrent et intègrent des périphrases. Il n'y a pas de correspondance lexicale, mais les informateurs reformulent librement le sens du terme et ils manifestent ainsi leur participation active (Castiglione, 2004, p. 59-60). Le phénomène est attesté aussi lors des entretiens effectués pour Seminario 2006 : pour siccità, une source de plus de 60 ans de Martina Franca fournit kwan:a y dza kjova 'quand il ne pleut pas' et deux informateurs âgés de Talsano proposent sejts ak:wa 'sans eau' (résultat enregistré à Talsano aussi dans Inchieste 2008).

\section{Conclusion}

Les objectifs de cette enquête étaient :

1) évaluer les dénominations d'un certain nombre de météoronymes dans la province de Tarente, non seulement d'un point de vue diatopique et sociolinguistique, mais aussi d'un point de vue synchronique;

2) vérifier les interférences entre le dialecte et l'italien, tout en considérant la condition particulière de l'interview et les problèmes causés par la technique de traduction.

Les analyses effectuées ont confirmé la validité de la différenciation, pour les facteurs internes, entre «concepts généraux» (froid, chaud, pluie, neige) et «concepts changeants» (pleuvioter, averse, etc.); cela avait déjà était souligné par Lazzari en 1919.

En effet, en ce qui concerne les concepts généraux (tels que pluie, grêle), d'un côté l'italien impose ses propres formes au détriment des formes endémiques de façon uniforme et généralisée, de l'autre côté, pour les phénomènes changeants et nuancés, il agit indirectement «destrutturando il campo di variazione lessicale (Sobrero et Miglietta, 2006, p. 164). Là où, dans le passé, différents types lexicaux étaient enregistrés pour le même phénomène (comme pour pleuvioter et averse), ceux-ci se réduisent considérablement et changent aussi qualitativement. Les formes dialectales, fortement marquées aux niveaux diatopique et diastratique, subissent un processus d'italianisation en générant des périphrases génériques. Des glissements de signification à la fois horizontaux (entre cohyponymes) et verticaux (d'hyponymes à hyperonymes et vice-versa) surviennent, ceux-ci sont donc utilisés par les locuteurs (surtout chez les jeunes) pour combler les vides lexicaux causés par la disparition progressive des types endémiques. Plus généralement, on acquiert à partir de l'italien des prêts (plus ou moins intégrés), qui visent à compenser la perte de 
règles de production de la part du dialecte (Dressler, 1988). Dans certains cas, «il contatto fra dialetto e italiano regionale è tanto stretto e continuo che i movimenti dall'uno all'altro delle varianti di uno stesso termine sono non solo paralleli e simmetrici, ma anche contrari e reciproci (sìccita $e$ siccità)» (Sobrero et Miglietta, 2006, p. 164).

De plus, à travers l'analyse des dénominations de foudre et tonnerre, nous avons remarqué comment les phénomènes liés sémantiquement par une grande proximité spatio-temporelle donnent lieu à des échanges lexicaux et plurifonctionnels. À l'origine de ces superpositions, de ce caractère «fuzzy» des résultats, se trouvent les conflits de rencontre entre systèmes linguistiques en contact (ibid.).

Des phénomènes d'hypertrophie synonymique et d'imprécision sémantique sont enregistrés pour rosée, bruine et gel. Tout cela doit être interprété à la lumière de faits extralinguistiques : dans le cas précis, la relation entre les expressions et les compétences des locuteurs d'un coté, et la réalité socio-économique de l'autre coté, est très forte. En effet, nous avons remarqué comment les phénomènes atmosphériques étaient bien distingués dans le passé, et comment ils le sont moins aujourd'hui, dans un contexte socio-économique très différent, non plus typiquement agricole, dans lequel la distinction exacte de rosée, bruine et gel n'est plus d'une importance fondamentale dans les vies des locuteurs. Nous remarquons que l'influence de l'italien est plus forte dans les centres les plus proches de la ville, centre dominateur, où le processus de standardisation apparaît accéléré, alors que dans les centres les plus éloignés de Tarente l'italianisation est plus lente, les centres sont connotés comme plus conservateurs. Ici on enregistre non seulement une tendance à conserver les types endémiques, mais aussi une poussée réactive qui a engendré une prolifération de termes synonymiques et polysémiques (cf. muttore, gelo/scilatura, brina). On pourrait supposer que, face à une période d'énormes bouleversements culturels, sociaux et économiques,

nelle aree a minore vocazione standardizzante l'incremento dei fenomeni di ambiguità, vaghezza, indeterminatezza, polisemia, sinonimia non sia mero cedimento strutturale ma, almeno in prima istanza, sia funzionale alla riorganizzazione del sistema più debole (nel nostro caso il dialetto), nella direzione di una maggiore flessibilità: flessibilità che, in ultima analisi, crea le condizioni per un adeguamento del sistema alle nuove esigenze semasiologiche. Un meccanismo ancora difensivo, insomma: il classico 'trasformarsi per sopravvivere' (Sobrero et Miglietta, 2006, p. 165).

Pour conclure, on peut affirmer que la province de Tarente montre des traits flagrants d'italianisation en ce qui concerne le niveau lexical - du moins dans le domaine des météoronymes - dans une situation qui n'est 
pas particulièrement conflictuelle avec la langue nationale. Dans un certain sens, c'est comme si le répertoire était triparti : l'italien standard d'un côté, le dialecte marqué de l'autre côté et une énorme zone intermédiaire caractérisée par la présence de phénomènes difficiles à classer justement à cause de leur caractère «fuzzy» propre à un système mixte, caractérisé par la polysémie, l'hypertrophie et l'imprécision sémantique.

\section{RÉFÉRENCES BIBLIOGRAPHIQUES}

Acquaviva Cosimo, 1931, Taranto... tarantina. Contributo allo studio delle tradizioni popolari, Tarente, Mazzolino, $205 \mathrm{p}$.

AIS = JABERG Karl et Jud Jakob, Sprach- und Sachatlas Italiens und der Südschweiz, 8 vol., Zofingen, Ringier, 1928-1910.

ALI $=$ Bartoli Matteo, Terracini Benvenuto, Vidossi Giuseppe, Grassi Corrado et Genre Arturo, Atlante Linguistico Italiano Rome, Poligrafico dello Stato (redazione presso l'Istituto dell'Atlante Linguistico Italiano, Università di Torino) (à paraître).

Bernardini-Marzolla Antonio, 1889, Saggio di un vocabolario domestico del dialetto leccese, Lecce, Spacciante, $101 \mathrm{p}$.

BerRuto Gaetano, 2001, «Parlare dialetto in Italia alle soglie del Duemila», dans G. L. Beccaria et C. Marello (éds), La parola al testo, 2 vol., Alexandrie, Dell'Orso, p. 33-49.

BotTigLIoni Gino, 1935, «Atlante linguistico-etnografico italiano della Corsica, promosso dalla R. Università di Cagliari. Introduzione», dans L'Italia dialettale, Arti grafiche Pacini Mariotti.

CAloro Antonio, 1982, «Espressioni dialettali salentine», dans Contributi, Rivista della Società di Storia Patria per la Puglia, sez. di Maglie, I, 3.

CANEPARI Luciano, 1980, Italiano standard e pronunce regionali, Padoue, CLEUP.

CAssano Giuseppe, 1935, Radeche vecchie. Proverbi, motti, frasi, indovinelli dialettali, credenze e giochi popolari tarantini, Tarente, Stab. Tip. F.lli Ruggieri.

Castiglione Marina, 2004, Traduzione e parlanti, l'esperienza dell'Atlante linguistico della Sicilia, Palerme, Centro di studi filologici e linguistici siciliani.

D'IPpolito Francesco, 1896, Vocabolario dialettale ossia il linguaggio vernacolo della provincia di Terra d'Otranto, Tarente, Tipografia del Commercio, $342 \mathrm{p}$.

De Mauro Tullio, 2001, Il dizionario della lingua italiana, Paravia.

De Noto Michele, 1897, Appunti difonetica sul dialetto di Taranto, Tarente, Vecchi, $39 \mathrm{p}$.

Devic L. Marcel, 1915, Dictionnaire étymologique des mots français d'origines orientales, Amsterdam, Oriental Press.

De Vincentis Domenico Ludovico, 1872, Vocabolario del dialetto tarantino in corrispondenza della lingua italiana, Tarente, Arnaldo Forni editore.

DISC = Dizionario Italiano Sabatini Coletti, Giunti, 1997. 
Dozy Reinhart et Engelmann Willem Herman, 1869, Glossaire des mots espagnoles et portugais dérivés de l'arabe, Leyden, E. J. Brill.

Dressler Wolfgang, 1988, «Language Death», dans F. J. Newmayer (éd.), Linguistics: The Cambridge Survey, vol. IV, Cambridge, Cambridge University Press, p. 184-192.

Enciclopedia L'Universale Scienze, Milan, 2003.

Gentile Tommaso, 1930, Nu stuezze di Viremijnze. Quadretti dialettali trentini, Tarente, Stab. Tip. Lodeserto, p. 99-110.

Giacalone Ramat Anna, 1984, «Aspetti del processo di sostituzione di lingua», dans G. B. Pellegrini, S. Bonato et A. Fabris (éds), Le isole linguistiche di origine germanica nell'Italia settentrionale, Roana, Istituto di Cultura Cimbra, p. 179-192.

Giacalone Ramat Anna, 1988, «L'interazione di fattori interni e di fattori esterni nella predicibilità del mutamento linguistico», dans V. Orioles (éd.), Modelli esplicativi della diacronia linguistica, Pise, Pacini, p. 167-184.

Gigante Nicola, 1986, Dizionario critico etimologico del dialetto tarantino, Manduria, P. Lacaita editore.

Gigante Nicola, 2002, Dizionario della parlata tarantina. Storico critico etimologico, Tarente, Mandese editore.

Gorgoni Giustiniano, 1891, Vocabolario agronomico con la scelta di voci di arti e mestieri attinenti all'agricoltura e col raffronto delle parole e dei modi di dire del dialetto della provincia di Lecce, Lecce, Spacciante, 515 p.

Grassi Giuseppe, 1925, Il dialetto di Martina Franca. Parte prima: Fonetica, Martina Franca, Aquazo \& Dragonetti, 92 p.

Greco Michele, 1935, Lu Mommu de Salice e il suo «Viaggio de Leuche a lengua noscia de Rusce», Lecce, La Modernissima.

JACOVelli E., 1952-1953, «Vocabolarietto di termini massafresi », dans L'ago nel Pagliaio, Mottola, Tip. Artistica Posa e Scapati.

LAZZARI Jady, 1919, I nomi di alcuni fenomeni atmosferici nei dialetti dell'Italia geografica, Pise, Mariotti.

Lокотsсн Karl, 1972, Etymol. Worterbuch de Europ. Worten Orient. Ursprungs, Heidelberg, Winter Verlag.

Meyer-LüBKe Wilhelm, 1968, Romanisches etymologisches Worterbuch (REW).

Miglietta Annarita, 1995, «I gradi dell'aggettivo», dans Bollettino dell'Atlante Linguistico Italiano, p. 79-105.

Miglietta Annarita, 2004, Fra dialetto e lingua in Salento, Lecce, Manni.

NADIR = Nuovo Atlante del Dialetto e dell'Italiano per Regioni, in corso di allestimento presso l'Università di Lecce.

Nobile Tommaso, 1999, Dizionario del dialetto ostunese, Galatina, Congedo.

Nunziato Alfredo, 1930, Zazarèddire (Cianfrusaglie), Tarente, Mandese.

Peluso Giacinto, 1982, Ricerche sul dialetto tarantino, Tarente, Soc. di Storia Patria per la Puglia.

Romanello Maria Teresa, «Sulla reazione della fonte», dans Dialetti e Lingue nazionali (Atti del XXVII Congresso della Società di Linguistica Italiana, Lecce, 28-30 octobre 1993), Rome, Bulzoni, p. 121-135. 
Sobrero Alberto. A. et Miglietta Annarita, 2001, «I tanti nomi della ceramica rustica in Salento: questioni di confine o vaghezza semantica?», dans Quaderni di Semantica, 1, p. 67-92.

Sobrero Alberto. A. et Miglietta Annarita, 2005, «Meteoronimi in Salento, fra dialetto e italiano», Plurilinguismo. Contatti di lingue e culture, no 12 , p. 145156.

Sobrero Alberto. A. et Miglietta Annarita, 2006, Lingua e Dialetto nell'Italia del Duemila, Galatina, Congedo editore.

Speziale G., 1940, Il dialetto di Martina Franca, Tesi di laurea, Rome.

STEIGER Arnald, 1932, «Contribución a la fonética del hispano-árabe y de los arabismos en el ibero-románico y el siciliano », dans Revista de filología española, Anejo XVII, Madrid.

Subak Julius, 1899, Das Zeitwort in der Mundart von Tarent, Jahresbericht der Kaiser-Franz-Joseph-Höheren-Handelsshule in Brünn, p. 21-43.

TAPPOLET Ernst, 1895, Die romanische Verwandschftsnamen, Strasbourg, Karl J. Trübner.

Terracini Benvenuto A., 1970, Lingua libera e libertà linguistica, Turin, Einaudi.

Torro Antonio, 1915, Vase e fiure, Padoue, Tip. Commerciale, 14 p. [Poesie in dialetto tarantino].

VDS = Rohlfs Gerhard, 1976, Vocabolario dei Dialetti Salentini (Terra d'Otranto), Galatina, Congedo (éd. originale 1959).

VIDos Bendict E., 1959, Manuale di Linguistica Romanza, Florence, Olschki.

Whorf Benjamin Lee, 1971, Language, Thought, and Reality, Cambridge, M.I.T. Press.

Zingarelli Nicola, 2005, Vocabolario della lingua italiana, Bologne, Zanichelli. 